\title{
Nonlinear higher-order spectral solution for a two-dimensional moving load on ice
}

\author{
FÉLICIEN BONNEFOY ${ }^{1} \dagger$, MICHAEL H. MEYLAN \\ AND PIERRE FERRAN T ${ }^{1}$ \\ ${ }^{1}$ Laboratoire de Mécanique des Fluides, Centrale Nantes, France \\ ${ }^{2}$ Department of Mathematics, The University of Auckland, New Zealand
}

We calculate the nonlinear response of an infinite ice sheet to a moving load in the time domain in two dimensions, using a higher-order spectral method. The nonlinearity is due to the moving boundary, as well as the nonlinear term in Bernoulli's equation and the elastic plate equation. We compare the nonlinear solution with the linear solution and with the nonlinear solution found by Parau \& Dias (J. Fluid Mech., vol. 460, 2002, pp. 281-305). We find good agreement with both solutions (with the correction of an error in the Parau \& Dias 2002 results) in the appropriate regimes. We also derive a solitary wavelike expression for the linear solution - close to but below the critical speed at which the phase speed has a minimum. Our model is carefully validated and used to investigate nonlinear effects. We focus in detail on the solution at a critical speed at which the linear response is infinite, and we show that the nonlinear solution remains bounded. We also establish that the inclusion of nonlinearities leads to significant new behaviour, which is not observed in the linear solution.

\section{Introduction}

The problem of a load moving over the surface of a continuous ice sheet floating on water of constant depth has been well studied. The book by Squire et al. (1996) describes in detail the research prior to 1996. One of the most straightforward problems is to determine the steady-wave pattern in the linear case for a point mass travelling at constant velocity. In this case the solution can be expressed in terms of a Fourier transform. The basis of this method was developed by Kheisin (1963), and Davys, Hosking \& Sneyd (1985) considered in detail the response for a steadily moving load point in three dimensions. Milinazzo, Shinbrot \& Evans (1995) also considered the steady-time problem in three dimensions, and they also considered more general load shapes and made extensive calculations and comparisons with experiments. The time-dependent solution was considered by Schulkes \& Sneyd (1988) for two dimensions and Nugroho et al. (1999) for three dimensions. Both solutions were for a steadily moving load starting from rest.

One of the most striking features of the solution for steady velocities is the existence of a critical speed, at which the response for the linear problem is infinite. At this speed the dispersion equation, which was first given by (Greenhill 1887), has a minimum, so that the group speed becomes zero in the frame of reference of the moving load, 
and energy is unable to propagate away from the moving load. Below and above the critical speed, the solution undergoes a qualitative change from an isolated depression to propagating waves in front and behind the point source. In the two-dimensional problem there also exists a second critical speed at the shallow-water wave speed. Since this critical speed is an artefact of the assumption of two-dimensionality, it is often not studied in detail.

Experimental measurements of loads moving on ice have been made, and the two most important are by Takizawa $(1985,1988)$ and Squire et al. (1988). They measured the response of the ice to a range of forcing, including those due to a moving vehicle and a low-flying aircraft. They found that there was good agreement between the linear theory and measurements, except at the critical speed. While the experimental solutions showed a sharp peak close to the critical speed, the solution remained finite.

Various theories have been proposed to understand better the solution close to the critical speed. Hosking, Sneyd \& Waugh (1988) considered a viscoelastic model for the ice and showed that under these assumptions the response is finite for all speeds. They also extended their viscoelastic model to the time-dependent case for a steadily moving load (Wang, Hosking \& Milinazzo 2004). Miles \& Sneyd (2003) considered the motion of an accelerating pressure (as opposed to one moving with constant velocity), and they solved it by using a Fourier transform in space and a Laplace transform in time. They showed that the solution remains bounded if accelerated through the critical speed. Recently Parau \& Dias (2002) (hereafter P\&D because of the importance of this paper to the present work) considered the nonlinear problem of a steadily moving load on ice. They transformed the problem to a dynamical system and were able to find solitary wave solutions, provided that the speed was close to the critical speed but less than it. We will discuss their paper in more detail later in the paper. Note that Schulkes \& Sneyd (1988) and P\&D considered only the two-dimensional problem.

A number of other nonlinear studies of floating plates, in the absence of a moving load, can be found in the literature. Forbes (1986) considered periodic finite-amplitude solutions for the propagation of waves under an elastic plate. Peake (2001) presented a study of a fluid-loaded elastic plate with a uniform mean flow in the supporting fluid. The nonlinear terms for the plate in these papers, and in P\&D, are all different. (The nonlinear terms in the fluid are the same.) There is no consensus over the correct nonlinear terms for a plate, and we consider here the simplest expression given by P\&D. However other nonlinear terms could easily be included in our formulation.

In the present paper we consider the nonlinear problem of the time-dependent motion of a moving load and show how the solution can be found using a modification of the higher-order spectral (HOS) method described in Bonnefoy et al. (2008). The latter has been implemented for both periodic domains (Ducrozet et al. 2007) and numerical wave tank configuration (Ducrozet et al. 2006). The HOS method was originally developed by Dommermuth \& Yue (1987) and West et al. (1987), to solve for nonlinear free-surface waves in the periodic configuration. This method uses a spectral expansion, coupled with the fast Fourier transform (FFT), to calculate the Dirichlet-to-Neumann map at the nonlinear free surface. The use of FFT provides a very good numerical efficiency that gives access to high-accuracy simulation on a large domain and/or during a long period of time. The method is ideally suited to the problem of moving loads on ice, as the ice deflection stands as a continuous function. It is however not intended to model discontinuous surfaces, such as ice floes and cracks.

The structure of the paper is as follows: In $\S 2$ we formulate the equations of motion and present a new non-dimensionalization. In $\S 3$ we rederive the linear solution in 
the time domain for constant velocity due to Schulkes \& Sneyd (1988), in a slightly different form. In $\S 4$ we derive the linear solution close to the critical speed and show that this has the form of a solitary wave of the kind found by P\&D for the nonlinear problem. We then consider a linearization of the solution of $P \& D$ and find that this is identical to our linear solution, once an error in the P\&D solution has been found. In $\S 5$ we show how the higher-order spectral method can be modified, to allow for the presence of the floating ice plate. We also present a perturbation solution which is valid for the static forcing case. In $\S 6$ we present a careful validation of the HOS solution by comparison to the static solution, the linear solution and the solution of $\mathrm{P} \& \mathrm{D}$. We then use the model to make various investigations, focussing on the critical velocity but also considering other situations and showing various nonlinear effects. Section 7 is a summary.

\section{Equations of motion}

We begin by presenting a derivation of the equations of motion. For the linear case, these have been presented many times before, and a detailed discussion of them can be found in Squire et al. (1996). The nonlinear terms which we include in our derivation are the same as those used in $\mathrm{P} \& \mathrm{D}$. The main difference in our presentation and those given previously is the choice of non-dimensionalization. We also present the derivation in two dimensions only, since this is the only situation for which we will make numerical calculations.

An elastic plate floats on the surface of an inviscid and irrotational fluid, which is infinite in the $x$ direction and has finite depth in the $z$ direction. The ice sheet covers the entire water surface which is at $z=\xi$, where $\xi$ is the deflection of the ice, and the bottom surface of the fluid is at $z=-h$. The fluid is governed by Laplace's equation with a no-flow condition at $z=-h$,

$$
\begin{aligned}
\Delta \phi & =0, \\
\partial_{z} \phi & =0, \quad z=-h,
\end{aligned}
$$

where $\phi$ is the velocity potential of the fluid. We include nonlinear effects from the plate equation and by including the exact deflection of the interface. The kinematic equation from the continuity of velocity is

$$
\xi_{t}=\phi_{n}, \quad z=\xi .
$$

We also have a dynamic equation from the pressure forces, which are given by the nonlinear Bernoulli equation, the nonlinear bending force of the plate and the external pressure applied to the plate:

$$
\rho \phi_{t}+\frac{1}{2} \rho|\nabla \phi|^{2}+\rho g \xi+D \partial_{x}^{2}\left(\frac{\partial_{x}^{2} \xi}{\left(1+\left|\partial_{x} \xi\right|^{2}\right)^{3 / 2}}\right)=-p(x, t), \quad z=\xi,
$$

where $\rho$ is the density of the plate; $D$ is the bending rigidity of the plate; and $p$ is the applied external pressure. Here we consider the nonlinear curvature of the plate, but we neglect both the finite thickness and the tension due to bending, following P\&D. Furthermore, we have set the inertia term of the plate to zero, also following P\&D. We note that the nonlinear expression for the plate is different in Forbes (1986) and Peake (2001). 


\subsection{Non-dimensionalization}

The equations are non-dimensionalized as follows: We scale the length $L=(D / \rho g)^{1 / 4}$, time by $T=\sqrt{L / g}$ and the mass by $\mathscr{M}=\rho L^{3}$. Under this non-dimensionalization the equations become

$$
\begin{aligned}
\frac{L^{2}}{T^{2}} \rho \bar{\phi}_{\bar{t}}+\frac{1}{2} \rho \frac{L^{2}}{T^{2}}|\nabla \bar{\phi}|^{2}+\rho g L \bar{\xi}+\frac{D}{L^{3}} \partial_{\bar{x}}^{2}\left(\frac{\partial_{\bar{x}}^{2} \bar{\xi}}{\left(1+\left|\partial_{\bar{x}} \bar{\xi}\right|^{2}\right)^{3 / 2}}\right) & =-\frac{\mathscr{M}}{L T^{2}} \bar{p}(\bar{x}, \bar{t}), \quad \bar{z}=\bar{\xi}, \\
\frac{L}{T} \bar{\xi}_{\bar{t}} & =\frac{L}{T} \bar{\phi}_{n}, \quad \bar{z}=\bar{\xi},
\end{aligned}
$$

and this simplifies to

$$
\begin{aligned}
\bar{\phi}_{\bar{t}}+\frac{1}{2}|\nabla \bar{\phi}|^{2}+\bar{\xi}+\partial_{\bar{x}}^{2}\left(\frac{\partial_{\bar{x}}^{2} \bar{\xi}}{\left(1+\left|\partial_{\bar{x}} \bar{\xi}\right|^{2}\right)^{3 / 2}}\right) & =-\bar{p}(\bar{x}, \bar{t}), \quad \bar{z}=\bar{\xi}, \\
\bar{\xi}_{\bar{t}} & =\bar{\phi}_{n}, \quad \bar{z}=\bar{\xi} .
\end{aligned}
$$

For the rest of the paper we will drop the overbar for clarity and use the nondimensional equations and variables. We believe that this non-dimensionalization is better than any used previously, because it leaves only the water depth and pressure as independent parameters.

\subsection{Moving load}

We consider the problem in which the pressure is given by a moving point load (sometimes called a line load since our problem is two-dimensional). We will assume that the load is given by a delta-function forcing. We could extend the theory to consider more complicated forces but do not do so to keep the presentation as simple as possible. We also assume that the point pressure is travelling at a constant speed $U$. This gives us $p(x, t)=\delta(x-U t) f(t)$, where $f$ is some arbitrary function. We then change coordinates to $x^{\prime}=x-U t$; so (2.4) becomes

$$
\begin{gathered}
\phi_{t}-U \partial_{x^{\prime}} \phi+\frac{1}{2}|\nabla \phi|^{2}+\xi+\partial_{x^{\prime}}^{2}\left(\frac{\partial_{x^{\prime}}^{2} \xi}{\left(1+\left|\partial_{x^{\prime}} \xi\right|^{2}\right)^{3 / 2}}\right)=-\delta\left(x^{\prime}\right) f(t), \quad z=\xi, \\
\xi_{t}-U \partial_{x^{\prime}} \xi=\phi_{n}, \quad z=\xi .
\end{gathered}
$$

Again, for clarity we will omit the prime for the rest of the paper.

\section{Linear solution}

The linear equations are given by the linearization of (2.5) and are of the following form:

$$
\begin{aligned}
\phi_{t} & =U \partial_{x} \phi-\partial_{x}^{4} \xi-\xi-\delta(x) f(t), \quad z=0, \\
\xi_{t} & =U \partial_{x} \xi+\phi_{n}, \quad z=0
\end{aligned}
$$

where $\phi_{n}=\partial_{z} \phi$ and with

$$
\begin{aligned}
\Delta \phi & =0, \\
\partial_{z} \phi & =0, \quad z=-h,
\end{aligned}
$$

where $h$ is the non-dimensional depth. We can solve this system of equations using the Fourier transform in the spatial variable, exactly as was done in Schulkes \& Sneyd 
(1988). We define

$$
\hat{\phi}(k, z, t)=\int_{-\infty}^{\infty} \phi(x, z, t) e^{\mathrm{i} k x} \mathrm{~d} x
$$

and likewise for $\hat{\xi}$. Equation (3.2) becomes

$$
\left.\hat{\phi}_{n}\right|_{z=0}=\left.k \tanh k h \hat{\phi}\right|_{z=0} .
$$

Substituting this into the Fourier transform of (3.1), we obtain

$$
\begin{aligned}
\hat{\phi}_{t} & =\mathrm{i} U k \hat{\phi}-\left(k^{4}+1\right) \hat{\xi}-f(t), \\
\hat{\xi}_{t} & =\mathrm{i} U k \hat{\xi}+k \tanh k h \hat{\phi} .
\end{aligned}
$$

This is a linear system of equations for $(\hat{\phi}, \hat{\xi})$, which we can write as

$$
\partial_{t}\left(\begin{array}{l}
\hat{\phi} \\
\hat{\xi}
\end{array}\right)-\left(\begin{array}{cc}
\mathrm{i} U k & -\left(k^{4}+1\right) \\
k \tanh k h & \mathrm{i} U k
\end{array}\right)\left(\begin{array}{l}
\hat{\phi} \\
\hat{\xi}
\end{array}\right)=\left(\begin{array}{c}
-f(t) \\
0
\end{array}\right) .
$$

This has the solution

$$
\left(\begin{array}{l}
\hat{\phi}(t) \\
\hat{\xi}(t)
\end{array}\right)=\int_{0}^{t} e^{\boldsymbol{A}(t-s)}\left(\begin{array}{c}
-f(s) \\
0
\end{array}\right) \mathrm{d} s+e^{\boldsymbol{A} t}\left(\begin{array}{l}
\hat{\phi}(0) \\
\hat{\xi}(0)
\end{array}\right),
$$

where $\boldsymbol{A}$ is the matrix

$$
\boldsymbol{A}=\left(\begin{array}{cc}
i U k & -\left(k^{4}+1\right) \\
k \tanh k h & \mathrm{i} U k
\end{array}\right)
$$

\subsection{Heaviside forcing}

We are especially interested in the case when $f(s)=p H(s)$, where $H$ is the Heaviside function, and when the plate is initially at rest. This is precisely the case considered by Schulkes \& Sneyd (1988). This gives us

$$
\begin{aligned}
\left(\begin{array}{c}
\hat{\phi}(t) \\
\hat{\xi}(t)
\end{array}\right) & =\int_{0}^{t} e^{\boldsymbol{A}(t-s)}\left(\begin{array}{c}
-p \\
0
\end{array}\right) \mathrm{d} s \\
& =\boldsymbol{A}^{-1}\left(e^{\boldsymbol{A} t}-I\right)\left(\begin{array}{c}
-p \\
0
\end{array}\right) .
\end{aligned}
$$

Note that an exact expressions for $e^{\mathbf{A} t}$ can be easily derived, and this can be found in Schulkes \& Sneyd (1988).

\subsection{Steady time limit}

Schulkes \& Sneyd (1988) show by very careful arguments that, except at two critical speeds (which will be discussed shortly), as $t \rightarrow \infty$ the inverse Fourier transform of $e^{\boldsymbol{A} t}$ tends to zero. This means that the long-time solution is given by

$$
\boldsymbol{A}^{-1}\left(\begin{array}{l}
p \\
0
\end{array}\right) \text {. }
$$

The steady problem can also be found by setting all time derivatives equal to zero in (3.1) and assuming that $f(t)=p$ (i.e. the pressure is a constant). We then obtain

$$
\begin{aligned}
\mathrm{i} U k \hat{\phi}-\left(k^{4}+1 \hat{\xi}-p\right. & =0, \\
\mathrm{i} U k \hat{\xi}+k \tanh k h \hat{\phi} & =0,
\end{aligned}
$$


which is nothing more than (3.3). Solving for the transform of the displacement we obtain

$$
\hat{\xi}=-\frac{p}{k^{4}+1-U^{2} k \operatorname{coth} k h},
$$

and the steady pattern can be found by inversion. The inversion needs to be performed with care, because of the singularities in the expression for $\hat{\xi}$ in (3.4). We calculate the inversion by the method described in Whitham (1974) for waves on a steady stream and further applied to floating ice by Milinazzo et al. (1995). We write the time dependence of the pressure as $p e^{-\sigma t}$ for $\sigma>0$ and then assume the same dependence for $\hat{\xi}$ and $\hat{\phi}$, so that $\hat{\phi}=\hat{\phi}^{\sigma} e^{-\sigma t}$ and $\hat{\xi}=\hat{\xi}^{\sigma} e^{-\sigma t}$. This gives us

$$
\begin{aligned}
-\sigma \hat{\phi}^{\sigma} & =\mathrm{i} U k \hat{\phi}^{\sigma}-\left(k^{4}+1\right) \hat{\xi}^{\sigma}-p, \\
-\sigma \hat{\xi} & =\mathrm{i} U k \hat{\xi}^{\sigma}+k \tanh k h \hat{\phi}^{\sigma},
\end{aligned}
$$

which has the solution

$$
\hat{\xi}^{\sigma}=-\frac{p}{k^{4}+1-\frac{(U k+\mathrm{i} \sigma)^{2}}{k} \operatorname{coth} k h} .
$$

We then take the limit as $\sigma \rightarrow 0$. The properties of this solution is discussed in detail in Schulkes \& Sneyd (1988). The properties of this solution for three dimensions are discussed in detail in Davys et al. (1985).

\subsection{The critical speed $c_{\min }$}

In two dimensions there exist two critical speeds at which the solution corresponding to (3.5) is unbounded. One speed is the shallow-water speed $U=\sqrt{h}$ and corresponds to the wavenumber $k=0$ in (3.5) and a uniform rise in the time domain. We do not consider this critical velocity because it does not appear in the three-dimensional solution or experimental measurements. Furthermore, the HOS method that we develop conserves volume and cannot be used to analyse this case. The second critical speed corresponds to a minimum in the phase speed and is discussed below.

The plane progressive waves propagating under an ice sheet satisfy the well-known dispersion equation

$$
\omega^{2}=\left(k^{5}+k\right) \tanh (k h)
$$

(Greenhill 1887). The phase velocity is given by

$$
c=\overline{\left(k^{3}+1 / k \tanh k h\right.} \text {. }
$$

A very important property of $c$ is the existence of a critical speed $c_{\min }$ at which there is a minimum in the phase velocity. We denote the wavenumber at the critical speed by $k_{\min }$ and note that it satisfies the following equation:

$$
\left(3 k_{\text {min }}^{2}-1 / k_{\text {min }}^{2} \tanh k_{\text {min }} h+\left(k_{\text {min }}^{2}+1 / k_{\text {min }} h \operatorname{sech}^{2}\left(k_{\text {min }} h\right)=0 .\right.\right.
$$

At the critical speed $c_{\min }$ the group velocity and the phase velocity are equal. It follows physically that if a moving load is travelling with $U=c_{\min }$, then energy will not be able to propagate relative to the load and will therefore accumulate. There is a great deal of discussion about the critical load speed in the papers of Schulkes \& Sneyd (1988) and P\&D amongst others. At this critical speed the linear solution for the point forcing becomes unbounded as $t \rightarrow \infty$, in both two and three dimensions. When measurements of moving loads have been made a sharp peak in the response is observed close to $c_{m i n}$, but the solution remains finite. Much of the focus of research 
in this area has been on understanding the behaviour near this critical point. We will return to this situation in the results section.

\section{Solution just below the critical speed}

We consider here in detail the solution for speeds just below the critical speed. In this case the solution for both the linear and nonlinear problems consists of a solitary wave which oscillates and decays. P\&D solved the same nonlinear equations as we have here for the case of speeds just below the critical speed, and that is the motivation for this section. We also consider an approximation of the linear solution close to the critical speed, which is not mentioned in previous works. This allows us to compare the solution of P\&D with the linear solution in the small-pressure limit. The reason for the inclusion of the information in this section in such detail is that it provides strong evidence that the theory developed in $\mathrm{P} \& \mathrm{D}$ is correct (once the error in their work is corrected).

\subsection{Solution for $U$ near $c_{\text {min }}$ for the linear case}

We consider the linear case in which

$$
U=c_{\min }(1-\beta), \quad \beta>0,
$$

and $\beta$ is small. We restrict ourselves here to the case of infinite depth, to keep the expression simple and because this is the only case we will compare with the results of P\&D. The general case of finite depth can easily be developed in the same way as for infinite depth. The equation for the displacement in infinite depth is

$$
\left.\xi=-\frac{p}{2 \pi} \int_{-\infty}^{\infty} \frac{e^{-\mathrm{i} k x} \mathrm{~d} k}{k^{4}+1-U^{2}|k|}=-\frac{p}{\pi} \operatorname{Re} \int_{0}^{\infty} \frac{e^{-\mathrm{i} k x} \mathrm{~d} k}{k^{4}+1-U^{2} k}\right] .
$$

We want to estimate this integral, using the residue theorem. We therefore need to solve the equation $k^{4}+1-U^{2} k=0$ with $U$ just below $c_{\min }$ and find the solution $k$ in the lower complex plane which is close to $k_{\text {min }}$. We therefore write $k=k_{\text {min }}+\mathrm{i} \delta$ and substitute this to obtain

$$
\left(k_{\min }+\mathrm{i} \delta\right)^{4}+1-c_{\min }^{2}(1-\beta)^{2}\left(k_{\min }+\mathrm{i} \delta\right)=0 .
$$

We then consider this equation in powers of $\delta$ and $\beta$. The zeroth-order equation is

$$
k_{\text {min }}^{4}+1-k_{\min } c_{\min }^{2}=0,
$$

which is satisfied from the definition of the phase velocity. The equation at the first order in $\delta$ is

$$
i \delta\left(4 k_{\min }^{3}-c_{\min }^{2}=0,\right.
$$

which is satisfied from the definition of $k_{\min }$. This gives us the following equation which is second-order in $\delta$ and first-order in $\beta$ (and has no product terms in $\delta$ and $\beta$ ):

$$
6 k_{\text {min }}^{2}(\mathrm{i} \delta)^{2}+2 c_{\text {min }}^{2} \beta k_{\text {min }}=0 .
$$

From this it follows that

$$
\delta= \pm \frac{\overline{c_{\min }^{2} \beta}}{3 k_{\min }}= \pm k_{\min } \quad \overline{\frac{4 \beta}{3}}
$$


We now need to determine the residue. We find that

$$
\begin{aligned}
\left.\frac{1}{4 k^{3}-U^{2}}\right|_{k=k_{\min }-\mathrm{i} \delta} & =\frac{1}{4\left(k_{\min }-\mathrm{i} \delta\right)^{3}-c_{\min }^{2}(1-\beta)^{2}} \\
& =\frac{1}{4 k_{\min }^{3}-12 k_{\min }^{2} i \delta+12 k_{\min } \mathrm{i}^{2} \delta^{2}-4 \mathrm{i}^{3} \delta^{3}-c_{\min }^{2}+2 c_{\min }^{2} \beta-c_{\min }^{2} \beta^{2}} \\
& \simeq \frac{-1}{12 k_{\min }^{2} \mathrm{i} \delta} .
\end{aligned}
$$

What this means is that close to $c_{\min }$ we have

$$
\left.\xi=-\frac{p}{6 k_{\min }^{2} \delta} e^{-\delta|x|} \cos k_{\min } x-\operatorname{Re} \frac{i p}{\pi} \int_{0}^{\infty} \frac{e^{-k|x|} \mathrm{d} k}{k^{4}+1+\mathrm{i} U^{2} k}\right] \quad \text { where } \delta=k_{\min } \quad \frac{\overline{4 \beta}}{3},
$$

where we have deformed the contour of integration in (4.1) from the real to the negative imaginary axis, and the solution for negative $x$ is found from symmetry (or by deforming the contour integration into the upper half-plane). Since integral in (4.2) is bounded, it becomes negligible in comparison to the other term as $\delta \rightarrow 0$ and $U \rightarrow c_{\min }$. This allows us to write

$$
\xi=-\frac{p}{6 k_{\min }^{2} \delta} e^{-\delta|x|} \cos k_{\min } x,
$$

which is an approximation to the linear solution for speeds close to (but below) $c_{\min }$. This approximation becomes relatively more accurate as the critical speed is approached.

\section{2. $P \& D$ solution}

P\&D presented a nonlinear solution in two dimensions for the steady motion of a moving load whose velocity is close to but below the critical load speed $c_{\min }$. Their method, which is only applicable in two dimensions, was based on transforming the equations so that the dependent variable was the velocity potential. They then transformed the equations of motion so that they obtained a nonlinear Schrödinger equation (NLS), with forcing to describe the wave envelope. They were able to construct solutions to this equation, some of which corresponded to the linear solution and its perturbation. We will summarize their results here which are of importance to us.

The key result of $\mathrm{P} \& \mathrm{D}$ is conveniently summarized on page 284 of their work. We will compare the solution given by (4.5) with the solution found by P\&D in the limit of small pressure. The key difficulty in making this comparison is the very different non-dimensionalization used in the two derivations. The solution derived by $\mathrm{P} \& \mathrm{D}$ is for the displacement (which we denote by $\widetilde{\xi}$ because of the different non-dimensionalization used) is

$$
\widetilde{\xi}(x)=-\frac{2}{k_{\min } \widetilde{L}} \quad \overline{\frac{2 \mu q_{1}}{\left|q_{2}\right|}}\left(\frac{1}{\sinh \left(\sqrt{\mu q_{1}} / \widetilde{L}|x|+\alpha\right)}\right) \cos \left(k_{\min } x\right),
$$

where $q_{1}$ and $q_{2}$ are constants which will be discussed shortly and $\widetilde{L}$ the length scale used by $\mathrm{P} \& \mathrm{D}$, and

$$
\frac{\cosh \alpha}{\sinh ^{2} \alpha}=\frac{\sqrt{\left|q_{2}\right|}}{2 \sqrt{2} q_{1}} \frac{\widetilde{\varepsilon}}{\mu}
$$


where $\widetilde{\varepsilon}$ is a term proportional to the pressure. We now consider the solution when $\widetilde{\varepsilon} \rightarrow 0$. Equation (4.8) has approximate solution

$$
2 e^{-\alpha}=\frac{\sqrt{\left|q_{2}\right|}}{2 \sqrt{2} q_{1}} \frac{\widetilde{\varepsilon}}{\mu}
$$

for small $\widetilde{\varepsilon}(\operatorname{large} \alpha)$. The equation for $\widetilde{\xi}$ is therefore

$$
\begin{aligned}
\widetilde{\xi}(x) & \simeq-\frac{2}{k_{\text {min }} \widetilde{L}} \quad \frac{\overline{2 \mu q_{1}}}{\left|q_{2}\right|} 2 e^{-\sqrt{\mu q_{1} / \widetilde{L}|x|-\alpha} \cos \left(k_{\text {min }} x\right)} \\
& =-\frac{2}{k_{\text {min }} \widetilde{L}} \quad \frac{2 \mu q_{1}}{\left|q_{2}\right|} \frac{\sqrt{\left|q_{2}\right|}}{2 \sqrt{2} q_{1}} \frac{\widetilde{\varepsilon}}{\mu} e^{-\sqrt{\mu q_{1} / \widetilde{L}|x|} \cos \left(k_{\min } x\right)} \\
& =-\frac{1}{k_{\text {min }} \widetilde{L}} \frac{\widetilde{\varepsilon}}{\sqrt{q_{1} \mu}} e^{-\sqrt{\mu q_{1} / \widetilde{L}|x|}} \cos \left(k_{\text {min }} x\right) .
\end{aligned}
$$

The form of (4.9) is almost identical to (4.6). All that remains is to substitute the values for $q_{1}$ and change the non-dimensionalization.

For the case of infinite depth $q_{1}=2^{1 / 3} / 3$. The length scale is $\widetilde{L}=U^{-2 / 3}$, and we assume that we are sufficiently close to the minimum speed that we can approximate $U \simeq c_{\text {min }}$. Note here that there is a misprint in the expression (4.14) for $\mu$ in $\mathrm{P} \& \mathrm{D}$ (confirmed by personal correspondence). The correct expression for $\mu$ should be $\mu=f-f^{*}$ (where $f$ and $f^{*}$ are defined in $\mathrm{P} \& \mathrm{D}$ ) not the incorrect expression give on page 291 of $\mathrm{P} \& \mathrm{D}$. We therefore obtain

$$
\begin{aligned}
\mu & =\frac{1}{c_{\text {min }}^{8 / 3}(1-\beta)^{8 / 3}}-\frac{1}{c_{\text {min }}^{8 / 3}} \\
& \sim \frac{8}{3} \beta c_{\text {min }}^{-8 / 3}
\end{aligned}
$$

for small $\beta$. For infinite depth we have simple expressions for $k_{\min }=3^{-1 / 4}$ and $c_{\text {min }}=\sqrt{k_{\text {min }}^{3}+1 / k_{\text {min }}}=2 / 3^{3 / 8}$. We then obtain

$$
\sqrt{q_{1} \mu} / \widetilde{L}=c_{\min }^{2 / 3} \quad \overline{\frac{8}{3} c_{\min }^{-8 / 3} q_{1} \beta}=k_{\min } \quad \frac{\overline{4 \beta}}{3}=\delta .
$$

Therefore by comparing (4.9) and (4.6), we can see that the decay lengths agree. We now consider the pressure term. The pressure non-dimensionalization is $U^{4 / 3}$ in two dimensions. From P\&D $\widetilde{\varepsilon}=p \mathscr{C}_{0} / c_{\text {min }}^{4 / 3}$ where $\mathscr{C}_{0}=4^{1 / 3} / 6$. Substituting these expressions into (4.6) and multiplying by $\widetilde{L}$ to transform to our dimensional form we obtain

$$
\begin{aligned}
\xi(x) & =-\frac{1}{k_{\min }} \frac{\widetilde{\varepsilon}}{\sqrt{q_{1} \mu}} e^{-\sqrt{\mu q_{1}} / \widetilde{L}|x|} \cos \left(k_{\min } x\right) \\
& =-\frac{1}{k_{\min }} \frac{\widetilde{\varepsilon}}{\widetilde{L} \delta} e^{-\delta|x|} \cos \left(k_{\min } x\right) \\
& =-\frac{p}{6 k_{\min }^{2} \delta} \frac{4^{1 / 3} k_{\min }}{c_{\text {min }}^{2 / 3}} e^{-\delta|x|} \cos \left(k_{\min } x\right) \\
& =-\frac{p}{6 k_{\min }^{2} \delta} e^{-\delta|x|} \cos \left(k_{\min } x\right) .
\end{aligned}
$$


We can therefore conclude that the solitary solution found by P\&D with small pressure forcing corresponds to the solution of the linear equations. We will make further comparison between the nonlinear solution presented below and the solitary solution of $\mathrm{P} \& \mathrm{D}$ (see $\S 6.1 .4)$.

\section{Nonlinear solutions}

\subsection{HOS method}

The main result of the present work is an extension of the HOS method to the case of a nonlinear floating plate. The HOS was developed independently by Dommermuth \& Yue (1987) and West et al. (1987) to simulate nonlinear free-surface waves. The HOS method uses a spectral expansion and the FFT coupled with a modified Taylor series expansion to derive a very efficient way of computing the Dirichlet-to-Neumann map on the free surface (which is not assumed to lie on $z=0$ as in the linear theory). The method is very efficient but not flexible, due to the requirement that all effects be written as an expansion about the free surface (so that a body which penetrated the fluid surface could not be considered). The method is ideally suited to the problem of a moving load on ice, because all motions can be expanded about the free surface. We will briefly describe the HOS method and present the modifications to the theory which are required to allow us to consider a floating ice sheet. It is worth mentioning here that the nonlinearity comes from both the nonlinear equations and the moving boundary.

We begin by defining the free-surface potential $\phi^{s}(x, t)=\phi(x, \xi, t)$ and the vertical velocity $W(x, t)=\partial_{z} \phi(x, \xi, t)$. (Note that these are defined on the moving boundary.) The nonlinear equations given by (2.5) now become

$$
\begin{aligned}
\partial_{t} \phi^{s}= & U \partial_{x} \phi^{s}-\frac{1}{2}\left|\partial_{x} \phi^{s}\right|^{2}-\xi+\frac{1}{2}\left(1+\left|\partial_{x} \xi\right|^{2}\right) W^{2} \\
& -\partial_{x}^{2}\left(\frac{\partial_{x}^{2} \xi}{\left(1+\left|\partial_{x} \xi\right|^{2}\right)^{3 / 2}}\right)-\delta(x) f(t), \quad z=\xi, \\
\partial_{t} \xi= & U \partial_{x} \xi+\left(1+\left|\partial_{x} \xi\right|^{2}\right) W-\partial_{x} \xi \partial_{x} \phi^{s}, \quad z=\xi .
\end{aligned}
$$

The HOS method requires that we restrict to a finite domain in the horizontal direction with length $L_{x}$ and that we apply a periodic condition at the horizontal boundaries. It is therefore straightforward to express $\xi$ and $\phi^{s}$ as a discrete Fourier expansion. We solve (5.1) and (5.2) in the time domain, assuming that we know $\phi^{s}$ and $\xi$ at time $t$. This means that we must calculate $W$, and this requires a solution of Laplace's equation subject to the domain defined by $\xi$ with surface potential given by $\phi^{s}$. The HOS is essentially a very computationally efficient method to calculate this mapping (the Dirichlet-to-Neumann map).

\subsubsection{HOS approximation for $W$}

The HOS method is based on a series expansion of $\phi$ and $W$ with respect to $\xi$ :

$$
\begin{gathered}
\phi(x, z, t)=\sum_{m=1}^{M} \phi^{(m)}(x, z, t), \\
W(x, t)=\sum_{m=1}^{M} W^{(m)}(x, t),
\end{gathered}
$$

where $M$ is the order of nonlinearity and $\phi^{(m)}$ and $W^{(m)}$ are quantities $O\left(\xi^{m}\right)$. We substitute this series into the definition of the surface velocity potential $\phi^{s}$ and perform 
a Taylor expansion of the potential $\phi$ about $z=0$. At the different orders $m$ one obtains

$$
\begin{aligned}
\phi^{(1)}(x, 0, t) & =\phi^{s}(x, t) \\
\phi^{(m)}(x, 0, t) & =-\sum_{k=1}^{m-1} \frac{\xi^{k}}{k !} \partial_{z}^{k} \phi^{(m-k)}(x, 0, t), \quad m=2, \ldots, M .
\end{aligned}
$$

The full Dirichlet problem for $\phi(x, z, t)$ on $z=\xi(x, t)$ can then be simplified into $M$ simpler Dirichlet problems on $z=0$, respectively for $\phi^{(m)}(x, z, t)$. These problems are successively solved by means of a spectral method using as a basis functions which satisfy Laplace's equation, the no-flow condition at the bottom and the periodicity conditions. Using this expansion, the potentials $\phi^{(m)}$ can be expressed as

$$
\phi^{(m)}(x, z, t)=\sum_{n=-N_{x}}^{N_{x}} A_{n}^{m}(t) f\left(k_{n} x\right) \frac{\cosh k_{n}(z+h)}{\cosh k_{n} h},
$$

where $f$ are the basis functions of the discrete Fourier transform of $\xi$ and $\phi^{s}$ and $k_{n}=2 n \pi / L_{x}$ is the wavenumber associated with the $n$th mode. Note that in the infinite depth case $\cosh k_{n}(z+h) / \cosh k_{n} h$ is replaced by $\exp \left|k_{n}\right| z$. The surface potentials $\phi^{(m)}$ are solved numerically in sequence following (5.3) and (5.4). The vertical velocity is then evaluated in sequence from the Taylor expansion

$$
W^{(m)}(x, t)=\sum_{k=0}^{m-1} \frac{\xi^{k}}{k !} \partial_{z}^{k+1} \phi^{(m-k)}(x, 0, t), \quad m=1 \text { to } M .
$$

The two formulations of Dommermuth \& Yue (1987) and West et al. (1987) are equal up to this point. However, we now use the formulation of West et al. (1987), which takes extra care to express the free-surface boundary conditions up to the order $M$ with respect to $\xi$. That is to say, if we denote by $W_{M}$ the sum $\sum_{m=1}^{M} W^{(m)}$, then we calculate the values of $\left(1+\left|\partial_{x} \xi\right|^{2}\right) W$ and $\left(1+\left|\partial_{x} \xi\right|^{2}\right) W^{2}$, using

$$
\begin{aligned}
& \left(1+\left|\partial_{x} \xi\right|^{2}\right) W=W_{M}+\left|\partial_{x} \xi\right|^{2} W_{M-2} \text { and } \\
& \left(1+\left|\partial_{x} \xi\right|^{2}\right) W^{2}=\left(W^{2}\right)_{M}+\left|\partial_{x} \xi\right|^{2}\left(W^{2}\right)_{M-2} .
\end{aligned}
$$

\subsubsection{Flexural rigidity}

The nonlinear flexural rigidity of the ice sheet is taken into account in the freesurface equation for $\partial_{t} \phi^{s}$ :

$$
\partial_{x}^{2}\left(\frac{\partial_{x}^{2} \xi}{\left(1+\left|\partial_{x} \xi\right|^{2}\right)^{3 / 2}}\right)=A \partial_{x}^{4} \xi+2 \partial_{x} A . \partial_{x}\left(\partial_{x}^{2} \xi+\partial_{x}^{2} A \partial_{x}^{2} \xi .\right.
$$

For consistency with the HOS method, the three curvature-related terms $A, \partial_{x} A$ and $\partial_{x}^{2} A$ are further expressed as Taylor expansions in $\xi$ as follows:

$$
\begin{aligned}
A & =1-\frac{3}{2}\left|\partial_{x} \xi\right|^{2}+\frac{15}{8}\left|\partial_{x} \xi\right|^{4}-\frac{35}{16}\left|\partial_{x} \xi\right|^{6} \ldots \\
\partial_{x} A & =B \partial_{x} \xi \partial_{x}^{2} \xi \\
B & =-3\left(1-\frac{5}{2}\left|\partial_{x} \xi\right|^{2}+\frac{35}{8}\left|\partial_{x} \xi\right|^{4}+\ldots\right),
\end{aligned}
$$




$$
\begin{aligned}
& \partial_{x}^{2} A=\partial_{x} B \partial_{x} \xi \partial_{x}^{2} \xi+B \partial_{x}\left(\partial_{x} \xi \partial_{x}^{2} \xi\right. \\
& \partial_{x} B=15\left(1-\frac{7}{2}\left|\partial_{x} \xi\right|^{2}+\ldots\right) \partial_{x} \xi \partial_{x}^{2} \xi .
\end{aligned}
$$

The order of these expansions is chosen so that the flexural term (5.5) is of the order $M$ in the dynamic boundary condition. For instance the expansions presented above are valid for $M=7$. Note that the linear case presented in $\S 3$ is recovered when we keep only the terms which are first-order in $\xi$, that is $A=1, \partial_{x} A=0$ and $\partial_{x}^{2} A=0$. The operators $\partial_{x}$ and $\partial_{x}^{2}$ are evaluated in Fourier space.

\subsubsection{Numerical absorption}

A pressure term is added to the free surface in order to absorb the outgoing waves that are generated by the moving load. Without this absorption the waves would be reflected due to the finiteness of the computational domain. The absorbing pressure is given by $p_{a}(x, t)=\rho v(x) \phi_{n}$ so that the corresponding power

$$
\mathscr{P}=-\int_{S} p_{a} \phi_{n} \mathrm{~d} S=-\rho \int_{S_{a}} \nu(x) \phi_{n}^{2} \mathrm{~d} S
$$

is always negative, provided that $v(x)$ is positive in the region $S_{a}$ in which there is absorption and zero elsewhere. Note that $p_{a}$ reduces to $p_{a}=\rho v(x) \partial_{t} \xi$ from the kinematic boundary condition. The damping function $v$ is chosen to be $v(x)=$ $v_{0} u^{2}(3-2 u)$ with $u=1-x / x_{a}$ on the left side of the domain and the symmetric extension with respect to $x=L_{x} / 2$ (to match the periodicity condition). The width of the damping area $x_{a}$ and its strength $v_{0}$ need to be tuned to the kind of expected deflection. We choose to set $v_{0}=2$ and vary $x_{a}$. For static deflection $\left(U<c_{\text {min }}\right)$, $x_{a}$ can be quite small, typically 10 times the characteristic length (which is 1 with our non-dimensionalization). For progressive waves $\left(U \geqslant c_{\min }\right)$, we need a larger absorbing area (up to 100 times the characteristic length) so that the transmitted, and more particularly the reflected, waves are correctly damped. The quality of the damping zone can be easily monitored by looking at the envelope of the travelling waves for a linear simulation ( $M$ set to unity). If a significant amount of energy is reflected the envelope will show an oscillatory pattern, whereas it will be flat when reflection is negligible.

\subsubsection{Time stepping}

The linear part of (5.1) and (5.2) is analytically integrated following the procedure described in $\S 3$, so that this part of the integration is exact. The nonlinear evolution of the system is integrated numerically using a fourth-order Runge-Kutta Cash-Karp scheme with adaptive time step size (Cash \& Karp 1990).

\subsubsection{Products and de-aliasing}

The nonlinear products involved in the free-surface boundary conditions (5.1) and (5.2) are computed in the physical space. This leads to the well-known aliasing phenomenon, which has to be addressed to preserve accuracy (see e.g. Canuto et al. 1988). In general, de-aliased computations can be obtained by using spectra extended with zero padding. The number of collocation points in the physical space is then chosen, to remove aliasing errors made on multiple products. The latter products are found in the free-surface boundary conditions on the one hand and within the iterative HOS solution for $W$ on the other hand. There are at most $M$ products (products involving $M$ terms). Applying the half rule, the number of points to use in 
the physical space $\left(N_{x}^{d}\right)$ to get a full de-aliasing is

$$
N_{x}^{d}=\frac{M+1}{2} N_{x}
$$

\subsubsection{Numerical details}

Forward speed and pressure can be imposed with any given time evolution. For some situations we require a ramp to smoothly increase from zero either the pressure or the velocity. In such cases, the ramp function $r(t)$ will be the third-order polynomial

$$
r(t)=\left(t / T_{r}\right)^{2}\left(3-2 t / T_{r}\right), \quad 0 \leqslant t \leqslant T_{r},
$$

where the ramp duration $T_{r}$ is chosen as appropriate. We also use a different notation, depending on whether the ramp is in velocity or pressure $\left(T_{r}^{U}\right.$ for velocity; $T_{r}^{P}$ for pressure).

Spatial derivatives are evaluated in Fourier space. In order to preserve coherency with the de-aliasing process, the differentiation is applied to wavenumbers $k_{n}$ up to $|n|=(M+1) N_{x} / 2$. Note that no sawtooth instability appears in this simulation (as it does in the free-surface case), and therefore no smoothing is either required or applied. In the time domain, the time step is controlled by a proportional feedback and the absolute error tolerance is fixed at $10^{-8}$. We will explicitly give the numerical parameters $M, N_{x}, L_{x}, T_{r}^{U}, T_{r}^{P}, v_{0}, x_{a}$ for every simulation.

\subsection{Static case}

A very simple perturbation solution can be developed when $U=0$. This will be useful to validate the HOS model with nonlinear flexural rigidity and check the numerical convergence (see $\S 6.1 .1$ ). With static ice deflection and null velocity, the kinematic free-surface condition reduces to zero, and the dynamic condition becomes

$$
\xi+\partial_{x x} \frac{\partial_{x x} \xi}{\left(1+\left(\partial_{x} \xi\right)^{2}\right)^{3 / 2}}=-p \delta(x) H(t) .
$$

Note that the water depth has no influence here. We expand the deflection in the perturbation series

$$
\xi=\varepsilon \xi_{1}+\cdots+\varepsilon^{5} \xi_{5}+O\left(\varepsilon^{6}\right),
$$

and the pressure is $p=\varepsilon p_{1}$. The linear equation is

$$
\left(1+\partial_{x x x x}\right) \xi_{1}=-p_{1} \delta(x),
$$

which can be solved for by the Fourier transform $(\S 3.2)$ to obtain

$$
\xi_{1}=-\frac{p_{1}}{2} \sin (|x| / \sqrt{2}+\pi / 4) e^{-|x| / \sqrt{2}} .
$$

At the second order, the dynamic condition gives $\left(1+\partial_{x x x x}\right) \xi_{2}=0$; so $\xi_{2}=0$. At the third order we get

$$
\left(1+\partial_{x x x x}\right) \xi_{3}=\frac{3}{2} \partial_{x x}\left(\partial_{x x} \xi_{1}\left(\partial_{x} \xi_{1}\right)^{2}\right)
$$

At the fourth order, again we get $\xi_{4}=0$, and at the fifth order, the dynamic condition is written

$$
\left.\left(1+\partial_{x x x x}\right) \xi_{5}=-\frac{3}{2} \partial_{x x} 2 \partial_{x x} \xi_{1} \partial_{x} \xi_{1} \partial_{x} \xi_{3}+\left(\partial_{x} \xi_{1}\right)^{2}\left(\partial_{x x} \xi_{3}-\frac{5}{4} \partial_{x x} \xi_{1}\left(\partial_{x} \xi_{1}\right)^{2}\right)\right] .
$$


Equations (5.7) and (5.8) are successively solved numerically as follows: The spatial domain of length $L_{x}$ is discretized into $q N$ points given by $x_{n}=n L_{x} /(q N)$. The Fourier domain is discretized over $N$ modes given by $k_{n}=n\left(2 \pi / L_{x}\right)$ with $n=-N / 2+1$ to $N / 2$. The $q$ factor is related to the de-aliasing of the products on the right-hand side of (5.7) and (5.8). For example the double product $\partial_{x x}\left(\partial_{x x} \xi_{1}\left(\partial_{x} \xi_{1}\right)^{2}\right)$ has $3 N$ Fourier modes. If a computation in the space domain over $N$ points is performed, then the high modes $|n|>N / 2$ fold onto the $|n| \leqslant N / 2$ first ones; this is the well-known aliasing phenomenon. A value $q=2$ ensures that the previous product is alias-free as the high modes (now $|n|>N$ ) fold onto the modes $|n|=N / 2+1$ to $N$, thus leaving intact the $|n| \leqslant N / 2$ ones. Practically, the product is evaluated in the space domain on $q N$ points, and the Fourier modes with $|n|$ above $N / 2$ are neglected (set to zero). A value $q=4$ is used in what follows to ensure alias-free products up to the fifth order. In the the expressions given later $\varepsilon^{n} \xi_{n}$ are replaced by $\xi_{n}$ for clarity. The values of $N, q, L_{x}, p$ will be specified later on.

\section{Results}

There are many possible results which we can present. However, we focus on quantifying the transition from linear to nonlinear behaviour and on the solution near $c_{\min }$. However, we begin with a careful validation of the HOS method.

\subsection{Validation}

Before going any further, it is necessary to validate the nonlinear HOS model we have developed. While the HOS nonlinear approach has been thoroughly validated for water waves (see e.g. Dommermuth \& Yue 1987 and West et al. 1987) the present application is new and needs to be checked.

\subsubsection{Static case}

Equation (3.4) gives the linear solution that we compare either with the HOS result for small pressure or with the linear HOS method which is obtained by setting $M=1$. The pressure is imposed at $t=0$ with a value $p=10^{-4}$ without any ramp, and the transient waves are absorbed by the numerical damping region. According to Schulkes \& Sneyd (1988) the steady state is approached relatively rapidly for low load speed, and we take the deflection at $t=600$ as an estimation of the steady state. Numerical parameters are set as follows: For the HOS model, domain length is set to $L_{x}=40$, and we vary $N_{x}$ to check grid convergence. In order to absorb the transients, the HOS model is run with an absorbing zone whose length $x_{a}$ is set to $10 \%$ of the domain length and a magnitude $v_{0}=2$. The perturbation solution is estimated with the same $L_{x}$ and pressure and with a varying $N=N_{x}$ and a fixed $q=4$ (see (§5.2)).

We estimate the relative error $\varepsilon_{1}$ as the mean quadratic error $\left\|\xi_{1}-\xi\right\|_{2}$ between linear theory $\xi_{1}$ in (5.6) and the linear HOS deflection $\xi(\mathrm{M}=1)$ divided by the maximum deflection of $\xi_{1}$. This relative error is given (in \%) in table 1. For comparison, we also give the magnitude $\mathscr{M}_{3}$ of the third-order deflection which we evaluate as the mean quadratic third-order elevation $\left\|\xi_{3}\right\|_{2}$ obtained from the perturbation theory (5.7) divided by the maximum deflection of $\varepsilon_{1}$ (given in $\%$ in Table 1). Table 1 shows that error in the linear deflection decreases when the number of modes is increased, as expected, until grid convergence is reached. The linear result given by the HOS model with $M=1$ therefore satisfactorily agrees with the linear theory presented in (§3.2) when $U=0$. Furthermore the deflection error obtained with the HOS model is four orders of magnitude below the third-order deflection; this very low level of error is encouraging for the accuracy of further nonlinear simulations. 


$\begin{array}{ccc}N_{x} & 10^{6} \times \varepsilon_{1} & 10^{2} \times \mathscr{M}_{3} \\ 16 & 8.4 & 0.27 \\ 32 & 6.3 & 2.0 \\ 64 & 2.2 & 1.8 \\ 128 & 1.3 & 1.3 \\ 160 & 1.2 & 1.2\end{array}$

TABLE 1. Error at first order (obtained with $M=1$ ).

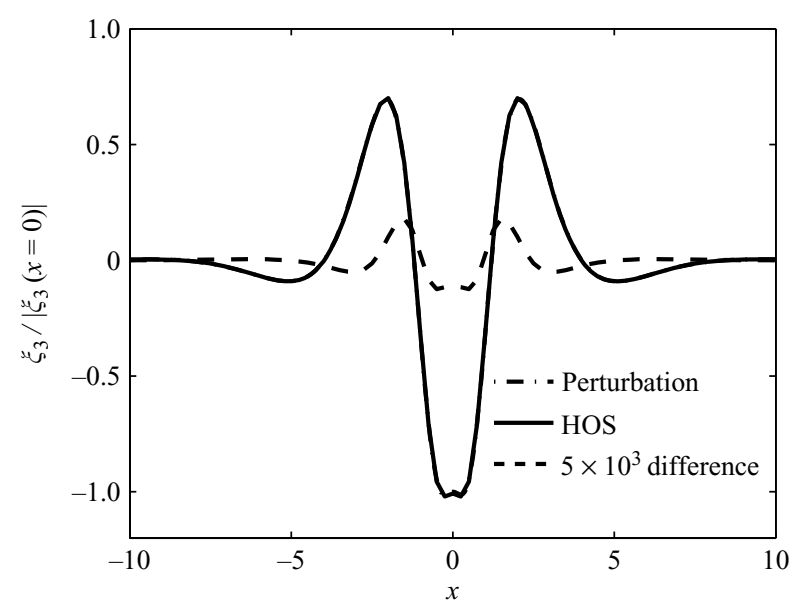

FIGURE 1. Third-order deflections.

Note that the constant component has been removed from both $\xi_{1}$ and the deflection from the HOS model before evaluating the error, as the HOS model ensures a constant volume of the numerical domain. This constant component could however still be recovered from the HOS model as $-\xi\left(x= \pm L_{x} / 2\right)$, provided that $L_{x}$ is long enough to ensure that oscillations are negligible at the boundaries.

We now investigate the nonlinear HOS and perturbation solution for the steady case. The input pressure is set to $p=1.5$, and the domain length is set to $L_{x}=40$. Nonlinear simulation are carried out with by varying $N_{x}$ and $M$. Again, the pressure is imposed at $t=0$ to be $p$, and there is no ramp. The deflection at $t=600$ is chosen, for comparison with the perturbation solution once the transient waves have been absorbed. The perturbation solution is estimated with $N=N_{x}$ and $q=4$. We have seen previously that a good agreement is obtained with the linear theory. We use this to estimate the nonlinear deflections at the third and fifth orders for the HOS model as $\xi-\xi_{1}$ and $\xi-\xi_{1}-\xi_{3}$ respectively, where $\xi$ is the nonlinear HOS results obtained with $N_{x}=160$ and $M=7$.

Figures 1 and 2 show a comparison between the third- and fifth-order deflections for both the perturbation model and the HOS model. We also plot on these figures the difference between the perturbation and HOS deflections. Clearly the perturbation results are correctly reproduced by the HOS model. In figure 1 the difference between the two models is the fifth-order deflection as can be clearly seen in figure 2. Similarly in figure 2, the difference between the fifth-order solutions should be the seventh-order deflection, but we did not develop the perturbation model of this order and cannot confirm this. 


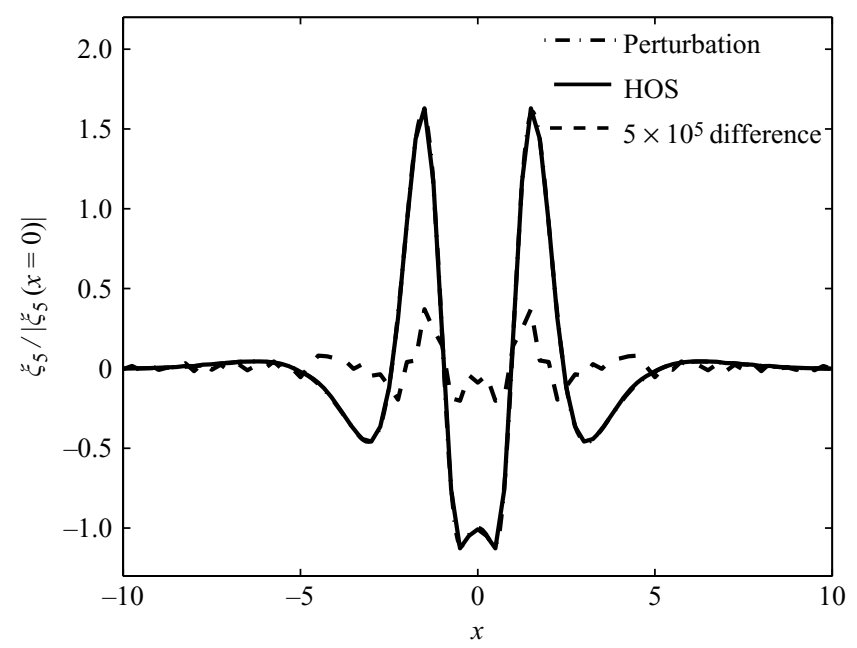

FiguRE 2. Fifth-order deflections.

$\begin{array}{cccc}N_{x} & M & 10^{2} \times \varepsilon_{3} & 10^{2} \times \mathscr{M}_{5} \\ 64 & 3 & 19 & 3.7 \\ 128 & 3 & 12 & 2.6 \\ 160 & 3 & 10 & 2.3 \\ 64 & 5 & 7.6 & 3.7 \\ 128 & 5 & 2.3 & 2.6 \\ 160 & 5 & 2.0 & 2.3 \\ 128 & 7 & 2.6 & 2.6 \\ 160 & 7 & 2.3 & 2.3\end{array}$

TABLE 2. Error at third order.

Table 2 shows the error $\varepsilon_{3}$ (in \%) between the HOS and perturbation solution at third order, evaluated as the mean quadratic error $\left\|\xi_{1}+\xi_{3}-\xi\right\|_{2}$ divided by the maximum deflection of $\xi_{3}$. We also give the magnitude $\mathscr{M}_{5}$ of the fifth-order elevation (in \%) in the last column, which is estimated as the mean quadratic fifth-order elevation $\left\|\xi_{5}\right\|_{2}$ divided by the maximum deflection of $\xi_{3}$. This relative error $\varepsilon_{3}$ tends towards $\mathscr{M}_{5}$ since the difference between the nonlinear deflection $\xi$ and the third-order deflection $\xi_{1}+\xi_{3}$ represents higher-order effects which are dominated by the fifthorder solution. However the results for $M=3$ show that the error $\varepsilon_{3}$ is greater than $\left\|\xi_{5}\right\|_{2}$. This means that $M=3$ is not strictly equivalent to the third-order perturbation solution. For $M \geqslant 3$ and $N_{x} \geqslant 128, \varepsilon_{3}$ is approximately equal to $\left\|\xi_{5}\right\|_{2}$, which means that grid convergence has been reached and that third-order effects are correctly reproduced. The validation made at the third order is enough for our purpose, and the HOS model is correct to this order for the nonlinear ice flexural rigidity.

\subsubsection{Linear subcritical case}

In the linear regime (3.4) provides the analytic deflection. This will be used to validate the linear part of the HOS model. We have also shown that this expression is equivalent to the solution presented by $\mathrm{P} \& \mathrm{D}$ in the small-pressure case, for subcritical velocities $\left(U<c_{\min }\right)$ close to $c_{\min }$. It is known that for non-static loads one may expect longer lasting transients (Schulkes \& Sneyd 1988), and we therefore use a ramp for 


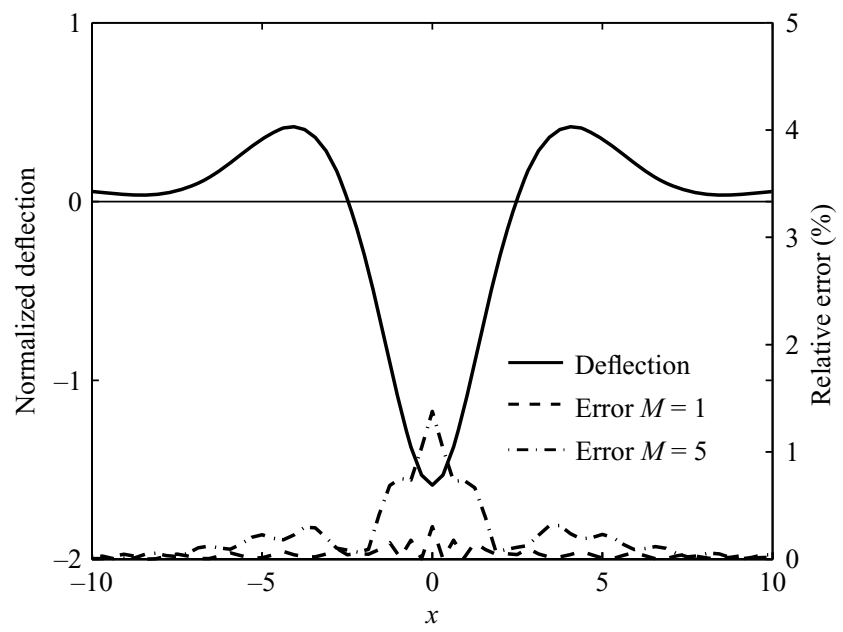

FIGURE 3. Linear deflection and errors at subcritical speed $U=1$.

the pressure. The $\operatorname{ramp} T_{r}^{P}=300$, and the simulation is run until $t=600$. This ensures that the transients have been absorbed by the lateral damping zone, so that $\xi$ is steady after the ramp. We consider the infinite water depth case. Figure 3 shows the linear deflection for $U=1$, after normalization by $p / 2 \sqrt{2}$ (the maximum deflection for $U=0$ ). The HOS model is run with both $M=1$ and $M=5$ for a small value of the pressure $\left(p=10^{-4}\right)$ and compared to the linear deflection given by (3.4). Once again, the constant mode has been removed from both the HOS and the linear solutions before comparison. Figure 3 shows the linear deflection (solid line) and the relative errors obtained with the two HOS simulations described above. We can see a good agreement between the HOS simulations and the linear theory. The HOS model will be further validated for subcritical velocities in the nonlinear regime (see $\S 6.1 .4$ ).

\subsubsection{Linear supercritical case}

Anticipating the nonlinear study that follows, the supercritical speed $U=1.38$ has been chosen so that the second-order wavenumbers do not correspond to either the linear left or the linear right wave field wavenumbers. The HOS deflection shown in figure 4 goes to zero at the boundaries of the domain due to the absorption zone. The fact that the envelope of the wave is constant outside of the damping zone $|x|<100$ is a good sign of the absorption quality. Figure 4 shows that the linear solution is correctly reproduced by the HOS model both with the linear version $(M=1)$ and with the nonlinear version with a small pressure $(M=5)$. This validates the model for supercritical velocity in the linear regime.

\subsubsection{Solution for $U$ near $c_{\text {min }}$ for the nonlinear case}

The nonlinear results are now compared with the solitary wave solution of P\&D, which is valid for speeds just below $c_{\min }$ (Note that $c_{\min } \approx 1.3246$ ). The coefficient $\varepsilon$ used to create figure $10(b)$ in $\mathrm{P} \& \mathrm{D}$ was set to 0.0497 instead of 0.003 , due to an incorrect non-dimensional form for the pressure forcing in their paper (personal communication with the authors). In two dimensions, the Dirac delta distribution is homogeneous to an inverse length and requires a non-dimensional form $L^{-1}$. This leads to a pressure scale of $\rho L^{3} T^{-2}$. 


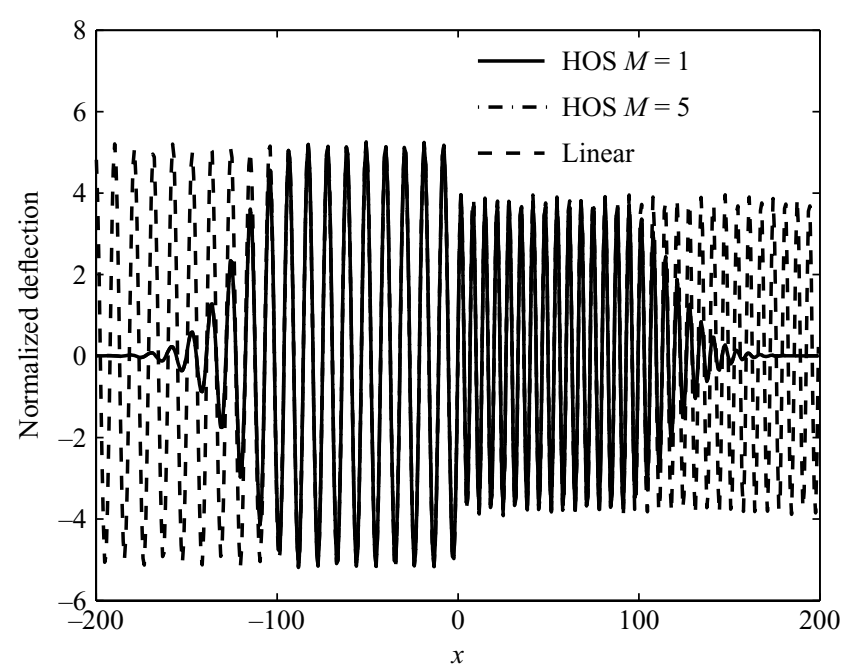

FIGURE 4. Linear deflection at supercritical speed $U=1.38$.

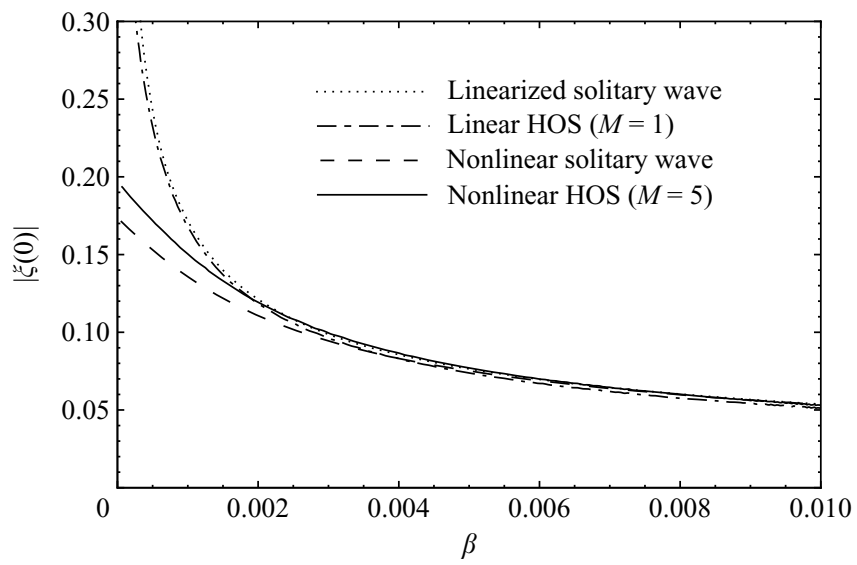

Figure 5. Comparison with the P\&D results $(\varepsilon=0.003)$.

The HOS model is run with both $M=1$ (linear) and $M=5$ (nonlinear) for $\varepsilon=0.003$. We let the velocity $U$ vary from 1.31 to 1.3246 by means of an appropriate ramp. It is important to slowly vary the velocity near $c_{m i n}$, since the deflection changes rapidly, and we choose $T_{r}^{U}=10^{5}$. Since $\varepsilon=0.003$ the pressure has to be adjusted at each velocity (because $p=\varepsilon / \mathscr{C}_{0} U^{4 / 3}$ ). Linear and nonlinear maximum deflection at $x=0$ obtained by both the solitary wave of P\&D and the HOS model are plotted in figure 5. We can see agreement between the linear deflections which tend to infinity as the velocity tends towards $c_{\text {min }}$. As was shown in $\S 4$, the linearized solitary solution tends to the linear solution given in Schulkes \& Sneyd (1988) as the integral part in (4.5) becomes negligible when $U$ tends towards $c_{\text {min }}$. (The linear HOS result includes this integral part.)

For values of $\beta$ greater than 0.005 we can see that the nonlinear effects are negligible, and the pressure imposed with $\varepsilon=0.003$ then falls into the linear regime. When we get 


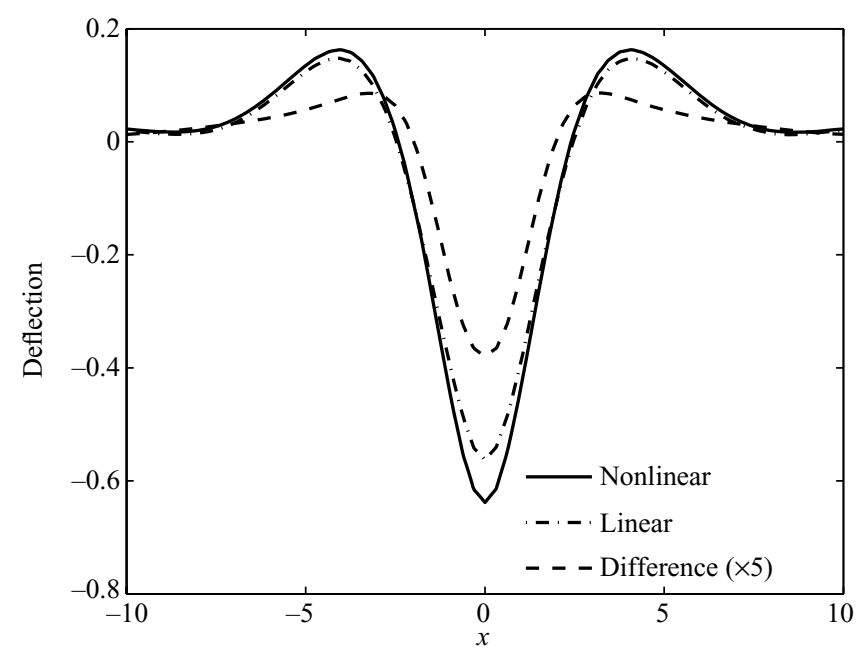

Figure 6. Nonlinear deflection at subcritical speed $U=1$.

closer to the critical velocity (lower $\beta$ ) the nonlinear deflection departs from the linear theory and we observe a finite deflection for $U=c_{\min }$. One may observe discrepancies between the HOS and NLS predictions: it appears that the NLS result under-predicts the maximum deflection. Similar results have been reported for capillary-gravity waves by Dias, Menasce \& Vanden Broeck (1996) and Laget \& Dias (1997). As the HOS computation with $M=3$ provides the same results, the third order of the NLS solution does not seem to be the source of this discrepancy. The narrow band assumption of the solitary solution might be a possible explanation.

\subsection{Nonlinear effects}

We now use the HOS method to investigate the finite-amplitude effects for significant pressures. We begin with the subcritical and supercritical regimes. To discuss the nonlinear effects, we extract the nonlinear components of the deflection by computing the difference between the linear case obtained with $M=1$ and the nonlinear case with $M=5$. This value of $M$ ensures that third-order effects are correctly reproduced.

\subsubsection{Subcritical speed}

In the first study we investigate the effect of finite pressure at subcritical speed. Longer ramps are required in this case in order to ensure the correct transient attenuation and that we have reached the steady state. The pressure time ramp is $T_{r}^{P}=3500$, and the simulation is run until $t=4000$.

As in the supercritical case (see $\S 6.2 .2$ ), the pressure ramp is set with $T_{r}^{P}=1000$, and simulation lasts until $t=2000$. Unless specified the nonlinear effects are studied for infinite water depth.

For moderate subcritical speeds, the nonlinear behaviour of the deflection is similar to that for the static case. Figures 6 and 7 show respectively the deflection in the space and in the Fourier domains for $U=1$ and a pressure $p=1$. The nonlinear effects are important near the moving load, where the maximum deflection occurs. For the pressure considered here the amplitude of the nonlinear effects reaches $12 \%$ of the total at $x=0$. In figure 7 the Fourier amplitude of the difference between linear and nonlinear defection shows a change of sign at $k=2.5$. A similar effect can be 


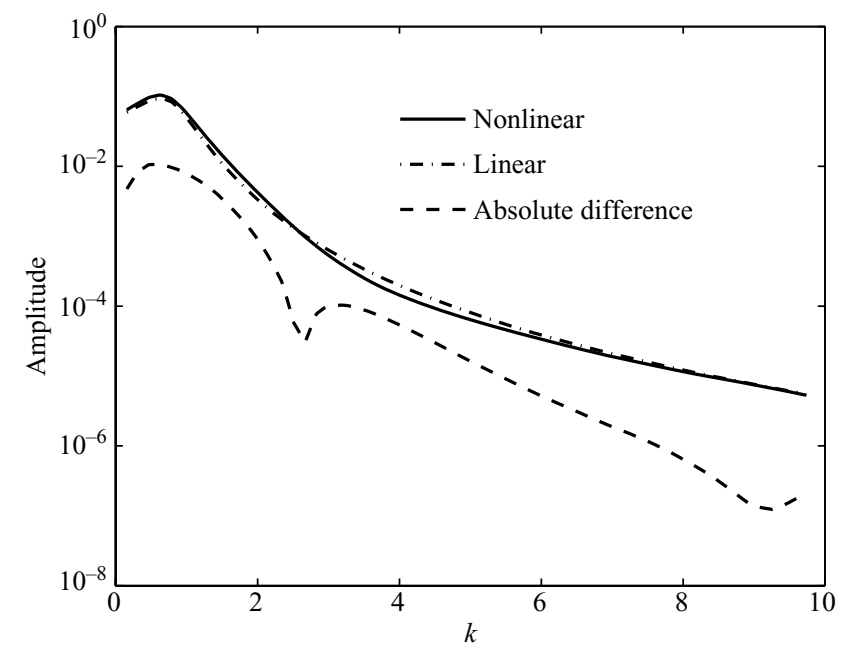

FIGURE 7. Fourier domain nonlinear deflection at subcritical speed $U=1$.

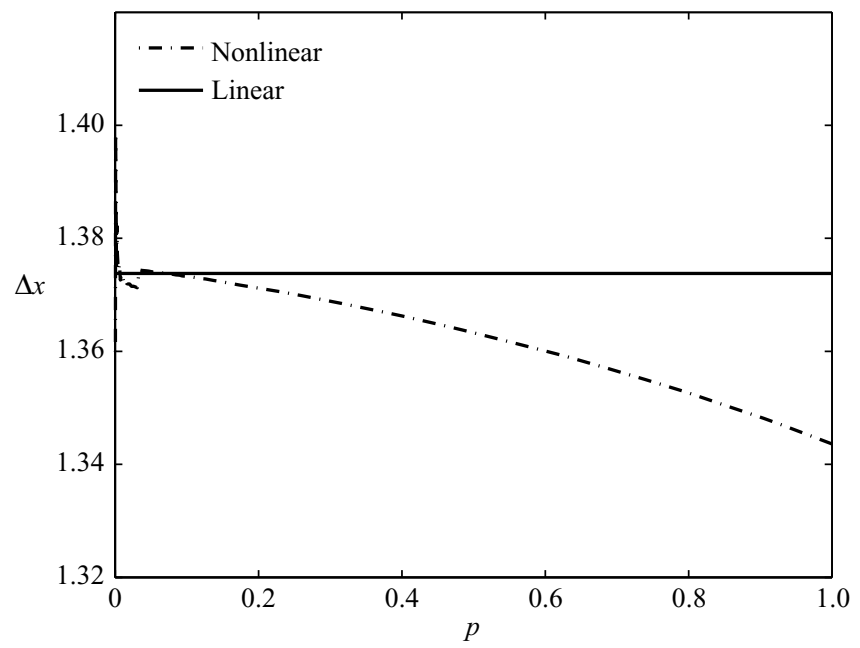

FIGURE 8. Full width of half maximum at subcritical speed $U=1$.

observed for static loads $(\S 6.1 .1)$. We have also checked that the high wavenumbers are correctly reproduced, by increasing the number of modes.

The full width of half maximum (FWHM) of the deflection is plotted against pressure in figure 8. One may observe that the nonlinear FWHM departs from the linear for non-zero pressures, and it can be seen that nonlinear effects tend to sharpen the deflection. Note that the reason why the FWHM estimation from the HOS is incorrect at very low pressures is the transients which have not been completely absorbed.

We now investigate the behaviour of the nonlinear solution when the velocity is less than the critical velocity. The velocity is slowly increased from 0.8 to 1.25 , so that the solution is quasi-static, for different pressures. The maximum deflection occurring at $x=0$ is plotted in figure 9. To validate the quasi-static assumption, we found the solution with a longer velocity ramp and checked that this solution was identical to 


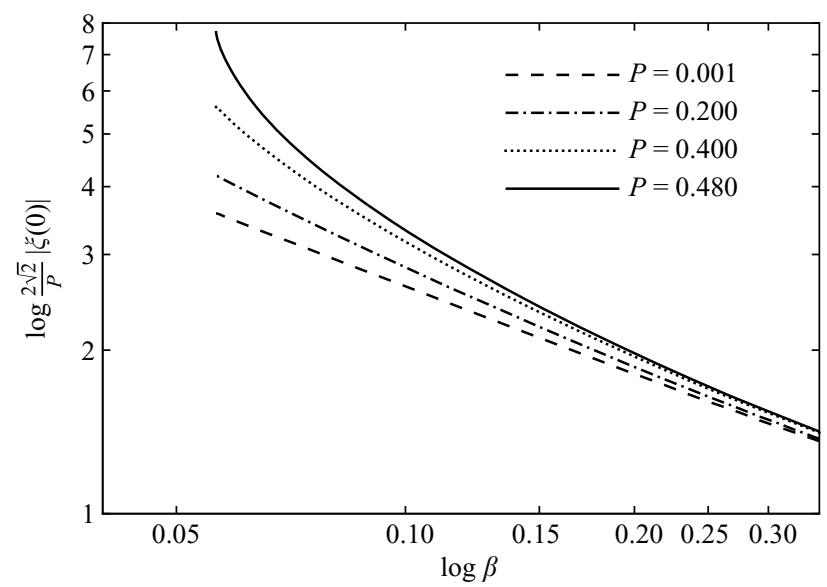

FIGURE 9. Maximum deflection against velocity for different pressures.

the one plotted in figure 9. The lowest pressure case (linear) clearly shows a one-half inverse power law $\beta^{-1 / 2}$ for the maximum deflection. When the pressure increases however, the maximum deflection departs from this law, and the closer the velocity becomes to the critical speed, the more pronounced the departure. With nonlinear effects taken into account one may observe normalized deflections more than twice as high as the linear prediction.

\subsubsection{Supercritical speed}

At supercritical velocities, one expects to see two progressive wave trains, one on each side of the pressure source. In order to investigate the nonlinear effects on these wave trains a useful separation of odd and even orders is made. Following Baldock, Swan \& Taylor (1996) we run two simulations with positive and negative pressures and denote $\xi^{+}$and $\xi^{-}$the corresponding deflections. By writing

$$
\xi_{\text {odd }}=\frac{1}{2}\left(\xi^{+}-\xi^{-}\right) \text {and } \xi_{\text {even }}=\frac{1}{2}\left(\xi^{+}+\xi^{-}\right)
$$

one may see that, as in a classical perturbation approach, odd and even orders of nonlinearities are effectively separated. The even nonlinear deflection $\xi_{\text {even }}$ is dominated by second-order effects.

Figure 10 shows the steady odd and even components of the nonlinear deflections for $p=0.08$. For comparison the linear deflection with positive pressure is added. The HOS results are obtained with $N_{x}=512$ modes in $L_{x}=200$ long domain at $t=2000$ after a ramp applied on pressure with $T_{r}^{P}=1000$ : the steady state is reached. Due to the progressive character of the generated waves, the absorbing zone must be efficient enough so that both the transmitted and reflected waves are correctly absorbed. Effective parameters are found to be $x_{a}=0.3 L_{x}$ and $\nu_{0}=0.2$. This corresponds to a smooth and long absorbing zone, compared to the associated wavelengths.

Behind the moving load in figure 10 we can see that the nonlinear wavelength is slightly larger than the corresponding linear one. In the fixed frame of reference, one would see a corresponding decrease in the frequency $f=U / \lambda$. The even nonlinear effects tend to lower both the crests and troughs of the trailing wave, in contrast to the usual Stokes waves. This effect was also reported for a moving disturbance on water without ice (Doctors \& Dagan 1980, e.g.). The deflection is modified by the relative phases of both components, which mainly depend on the velocity. The even 


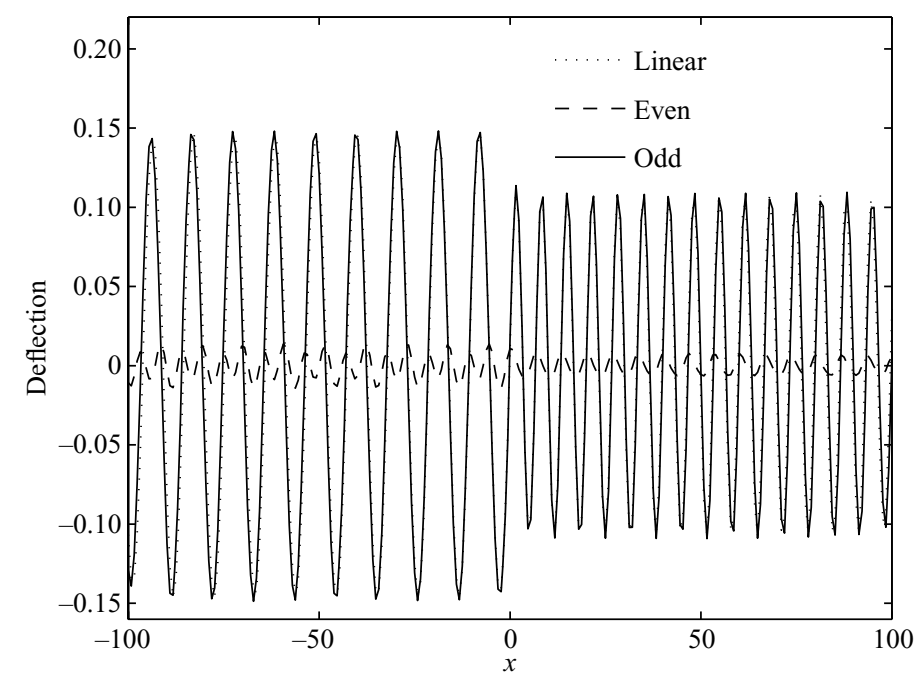

FIGURE 10. Nonlinear deflection at supercritical speed $U=1.38$.

deflection reaches here $10 \%$ of the total deflection. Ahead of the moving load one may observe that the even deflection mainly oscillates with the same wavelength as the linear deflection, and the finite amplitude effects tend to increase the wavelength.

Simulations at $U=1.45$ (which we do not plot) show stronger nonlinear effects for the same pressure $p=0.08$. Also, for this velocity, the even nonlinear effects tend to sharpen the crests and flatten the troughs in the trailing wave, as for typical water waves, while the observed wavelength still increases. The leading wave has an even deflection that oscillates with linear wavenumber as seen for $U=1.38$.

\subsection{Critical speed}

In the linear case, the solution at the critical speed exhibits a divergent behaviour. However, completely different wave patterns are obtained when the nonlinear terms are retained. The pressure is set to $p=0.025$ and the velocity to $U=c_{\min }$. Calculations are run until $t=3 \times 10^{4}$ over 4096 modes with the HOS nonlinearity parameter set to $M=5$. The domain is $L_{x}=4000$ long, and the absorption parameters set to $x_{a}=0.2 L_{x}$ and $v_{0}=0.005$ to ensure a correct damping. The displacement oscillates rapidly, and we plot the displacement envelope.

The deflection Hilbert transform is computed to obtain the envelope which is plotted in figures 11 and 12 at different instants. The deflection is normalized following Schulkes \& Sneyd (1988) with respect to vertical length scale

$$
L_{v}=\frac{2 p \tanh k_{\text {min }} h}{k_{\text {min }} c_{\text {min }} \partial^{2} c / \partial k^{2}\left(k_{\text {min }}\right) \sqrt{t_{\text {min }}}},
$$

where the growth timescale $t_{\min }$ is given by

$$
t_{\text {min }}=\frac{2 \pi}{k_{\text {min }}^{2} \partial c_{g} / \partial k\left(k_{\min }\right)} .
$$

At $t=30000$ the envelope has reached a steady state for $|x| \leqslant 1000$, and the corresponding displacement envelope is shown in figure 11. The far-field envelope is almost constant. The maximum amplitude is reached at $x=0$ and is 27 . The normalization parameter is $L_{v} \simeq 1 / 107$ which gives $|\xi(0)|=0.25$. The corresponding local steepness may be estimated as $k_{\min }|\xi(0)|=0.1$. 


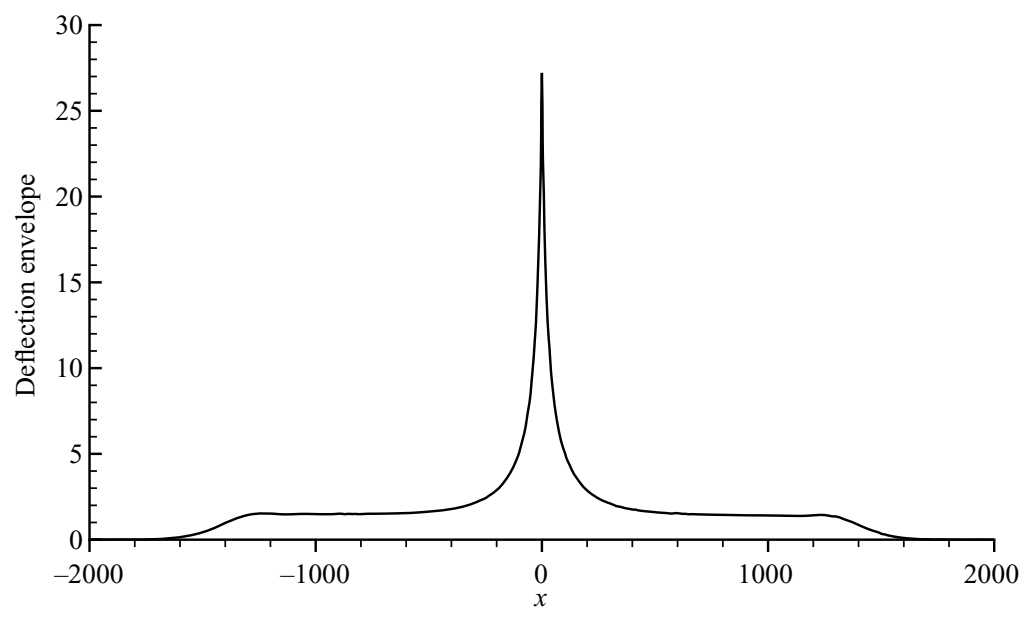

FIGURE 11. Nonlinear steady deflection at critical speed $U=c_{\min }$ and $t=30000$.

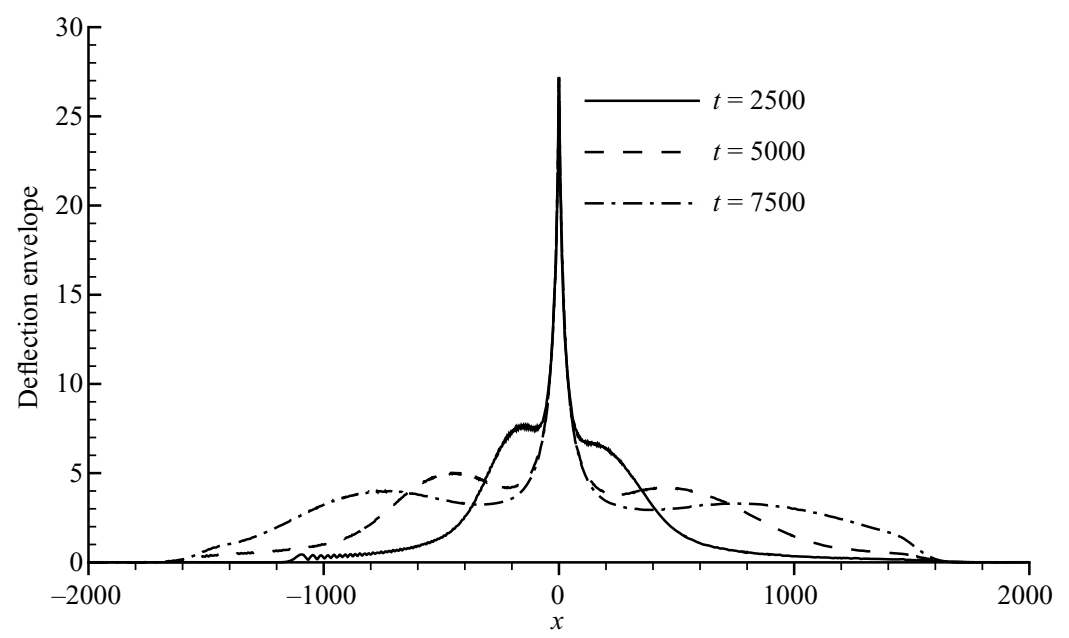

FIGURE 12. Evolution of the nonlinear deflection at critical speed $U=c_{\min }$.

Figure 12 shows the long-time evolution of the envelope. (The short-time behaviour is investigate in figure 13.) One can observe the emission of two wave packets propagating away from the pressure distribution. The deflection shows different leading and trailing waves, and the two wave packets are not symmetric.

Figure 13 shows the evolution of the maximum deflection at $x=0$ for both the linear and nonlinear case in a logarithmic scale. The linear maximum is evolving like $t^{1 / 2}$ as expected from the theory (Schulkes \& Sneyd 1988). The early evolution of the nonlinear case shows that the maximum deflection at first follows the same evolution until $t=500$, when the behaviour starts to differ from the linear one, and the maximum reaches a steady state near $t=2000$. Due to wave propagation, the steady state for $x \neq 0$ will be reached at later times as is shown in figure 12 .

\subsection{Experimental values}

Squire et al. (1988) conducted experiments on Antarctic Sea ice, using strain wave gauges, and they used vehicles and aircraft as the moving load. Obviously those 


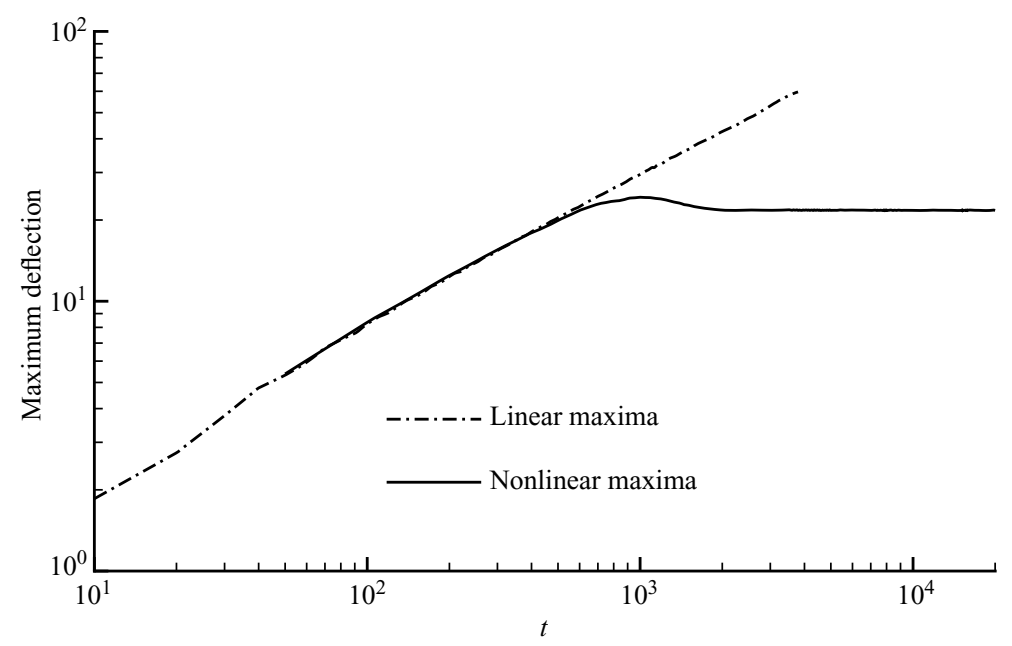

FiguRE 13. Evolution of the deflection at critical speed $U=c_{\min }$.

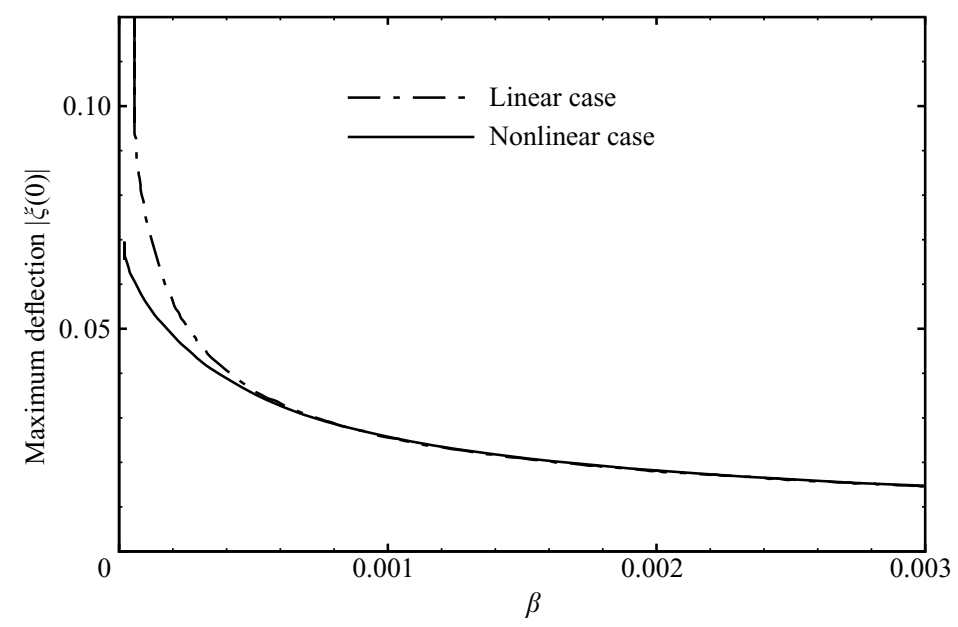

FIGURE 14. Maximum deflection approaching the critical speed.

experiments were three-dimensional, and any comparison with two-dimensional theory will only be an approximation. We consider the experiments conducted with the moving vehicle and set the pressure to be $p=\mathscr{M} /(\rho L 2 b)$, where $\mathscr{M}$ is the mass of the vehicle, $b$ its width. This corresponds to an infinite line of vehicles with zero spacing, and we expect this will be an over-prediction of the importance of nonlinear effects. The mass is assumed to be $\mathscr{M}=2100 \mathrm{~kg}$, and the width is assumed to be roughly $b=2 \mathrm{~m}$. The water depth is supposed to be infinite. The non-dimensional pressure is $p=0.0025, L_{x}=2000$ and $T_{r}^{U}=1.2 \times 105$. The experimental pressure is very small for the rigidity of the ice sheet, so that nonlinear effects will be of importance only near the critical speed. We therefore consider velocity in the range 1.315 to 1.32465. Other numerical parameters are $M=3, N_{x}=1024, x_{a}=500, v_{0}=0.6$. Figure 14 shows the maximum deflection $|\xi(0)|$ versus the factor $\beta$ already used in previous sections. Note that $\beta$ is much smaller than in figure 5, as the nonlinearities are much smaller. (The pressure for the experiments is eight times smaller than the 


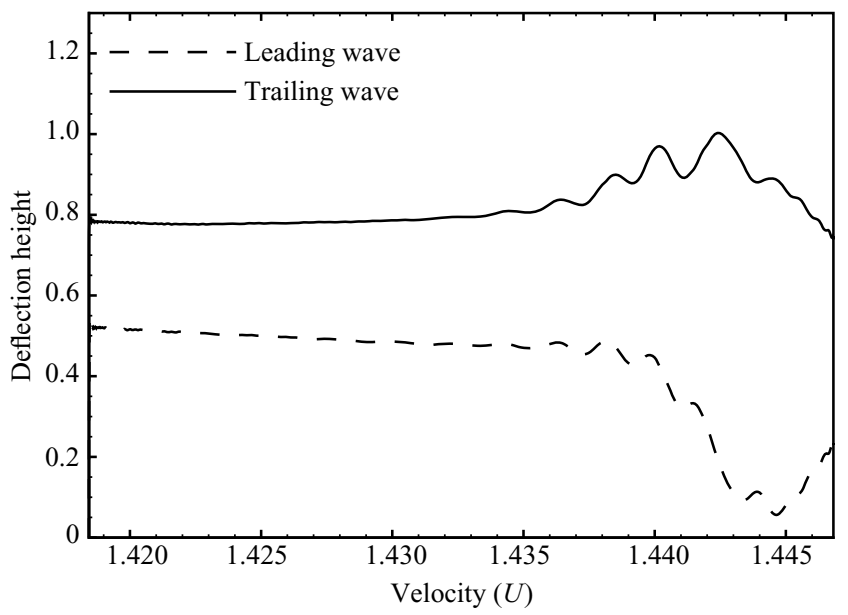

Figure 15. Evolution of the wave height near jump.

pressure used in $\S 6.1 .4$, as $p=\varepsilon / \mathscr{C}_{0} U^{4 / 3}$ means $p=0.02$ for $U=c_{\text {min }}$.) Furthermore the nonlinear amplitude is also smaller at the critical velocity when $\beta=0$ than in figure 5. Physically this amplitude corresponds to approximately $1.4 \mathrm{~m}$, which is far greater than was ever observed. However, it is important to note that this is the two-dimensional solution with a line load taken from the car width. It is probably much more reasonable to take a larger width to approximate a single car, and in this it could easily reduce the displacement by an order of magnitude. Furthermore, the long time limit was never reached in the experiment. Our computations show that after 1 minute the amplitude is $0.11 \mathrm{~m}$, and after 1 hour it is $0.95 \mathrm{~m}$. A fuller comparison requires a three-dimensional nonlinear solution.

\subsection{Further nonlinear effects}

For the problem of moving loads on ice, the linear theory does a satisfactory job of explaining the experimental observations except close to the critical velocity. We have therefore focused our attention on solutions near the critical velocity. Other nonlinear effects can be observed, and we present one such example here.

Figure 15 shows the deflection height estimated from the nonlinear leading and trailing wave as the velocity is slowly increased from 1.42 to 1.447 . The other numerical parameters for this simulation are $L_{x}=2000, M=3, N_{x}=2048, x_{a}=500, v_{0}=0.05$ and $p=0.0025$. Note that the load was conveniently set at $x_{l}=180$ in this simulation so that the trailing domain in which interesting features occur is longer. The leading and trailing wave trains undergo strong and asymmetric modification at the velocity around $U_{j}=1.442$. The nonlinear solution seems to jump from one branch of solution to another. Figures 16 and 17 show the simulated deflection respectively at $U=1.42$ and $U=1.447$, that is before and after the jump velocity $U_{j}$. Both cases present a leading wave whose envelope is uniform and symmetric with respect to $z=0$; however the trailing wave is asymmetric. Before the jump, the trailing envelope is uniform, and the depth of the troughs is more than the height of the crests, similar to what was observed in $\S 6.2 .2$. After the jump, figure 17 shows that the envelope is no longer uniform, and it attenuates away form the load. Observations in the Fourier domain reveal that the jump happens when the leading wavenumber $k_{f}$ is half the trailing wavenumber $k_{t}$, which shows that this phenomenon is associated with a triad interaction in which $k_{t}=k_{f}-k_{t}$ in the vicinity of the moving load. 


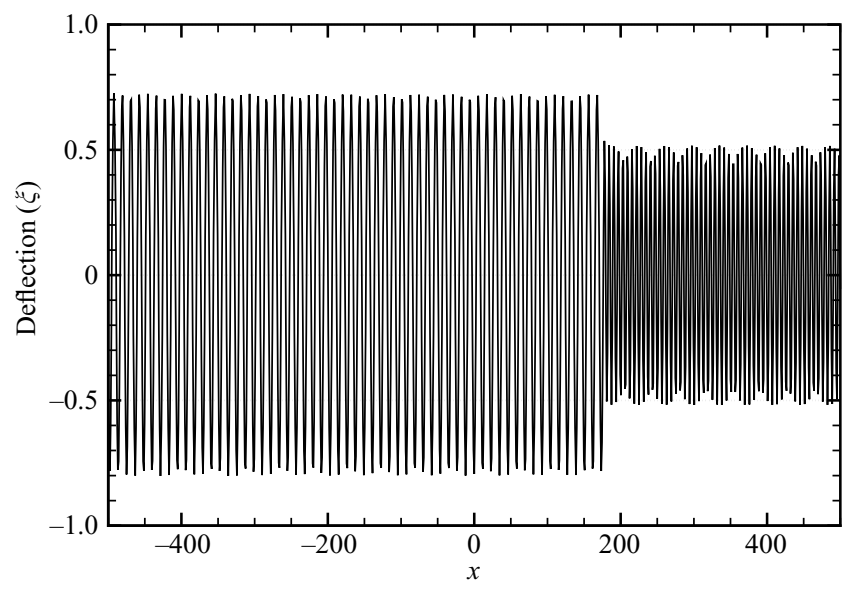

Figure 16. Wave field at $U=1.42<U_{j}$.

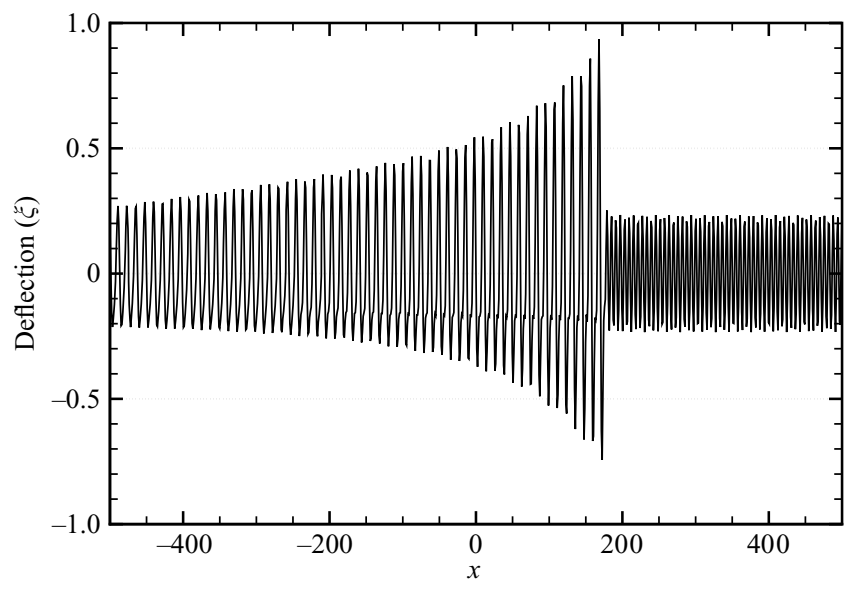

FIGURE 17. Wave field at $U=1.447>U_{j}$.

Similar effects were previously observed for water waves. Dias et al. (1996) investigated the case of capillary-gravity waves with finite amplitude. Two families of smallamplitude solitary waves are known to exist, bifurcating either from a uniform flow or from infinitesimal periodic waves at the minimum velocity. They were able to show that these two families were connected. They also found new families of solitary waves with finite amplitudes, similar to the one observed here. Laget \& Dias (1997) showed analogous results for capillary-gravity interfacial waves and found new families for large density ratios and finite amplitude. Here at fixed flexural rigidity and pressure, infinite depth and when the velocity is slowly varied, we found that the wave solution undergoes strong modifications, probably jumping from one family to another.

\section{Summary}

The aim of the present work was to investigate the effect of including nonlinear terms in the solution for a moving load on ice. We know that the linear theory gives good results when the load is small as has been shown by the experimental results, 
except close to the critical velocity. We have shown that for the typical loadings the nonlinear effects give a small correction to the linear solution. We have also validated the solution in P\&D (with a small correction) both by our HOS method and by a derivation from the linear solution. We have shown that the nonlinearities ensure that the solution remains bounded as the velocity approaches the critical velocity, although our two-dimensional model appears to over-predict the response (although we could scale the solution differently). Finally, we have shown that the nonlinear solution exhibits other nonlinear behaviour such as a triad interaction, which leads to a new type of solution not observed in the linear case.

\section{REFERENCES}

BAldock, T., Swan, C. \& TAYloR, P. 1996 A laboratory study of nonlinear surface waves on water. Phil. Trans. R. Soc. Lond. A 354, 649-676.

Bonnefoy, F., Ducrozet, G., Le Touzé, D. \& Ferrant, P. 2008 Advances in Numerical Simulation of Nonlinear Water Waves, chap. Time Domain Simulation of Non Linear Water Waves using Spectral Methods. World Scientific, submitted.

Canuto, C., Hussaini, M. Y., Quarteroni, A. \& Zang, T. A. 1988 Spectral methods in fluid dynamics. Springer series in comp. phys. 3-540-52205-0. Springer-Verlag.

CASH, J. R. \& KARP, A. H. 1990 A variable order Runge-Kutta method for initial value problems with rapidly varying right-hand sides. ACM Trans. Math. Softw. 16 (3), 201-222.

Davys, J. W., Hosking, R. J. \& Sneyd, A. D. 1985 Waves due to a steadily moving source on a floating ice plate. J. Fluid Mech. 158, 269-287.

Dias, F., Menasce, D. \& Vanden Broeck, J. M. 1996 Numerical study of capillary-gravity solitary waves. Eur. J. Mech. B/Fluids 15 (1), 17-36.

Doctors, L. G. \& DaGAN, G. 1980 Comparison of nonlinear wave-resistance theories for a twodimensional pressure distribution. J. Fluid Mech. 98, 647-672.

Dommermuth, D. G. \& Yue, D. K. 1987 A high-order spectral method for the study of nonlinear gravity waves. J. Fluid Mech. 184, 267-288.

Ducrozet, G., Bonnefoy, F., Le Touzé, D. \& Ferrant, P. 2006 Implementation and validation of nonlinear wave maker models in a HOS Numerical Wave Tank. Int. J. Offshore Polar Eng. 16 (3), 161-167.

Ducrozet, G., Bonnefoy, F., Le Touzé, D. \& Ferrant, P. 2007 3-D HOS simulations of extreme waves in open seas. Nat. Hazards Earth Syst. Sci. 7, 11-14.

Forbes, L. K. 1986 Surface waves of large amplitude beneath an elastic sheet. part 1. high-order series solution. J. Fluid Mech. 169, 409-428.

Greenhill, A. 1887 Wave motion in hydrodynamics. Am. J. Maths 9, 62-112.

Hosking, R. J., SNeYd, A. D. \& WaUgh, D. W. 1988 Viscoelastic response of a floating ice plate to a steadily moving load. J. Fluid Mech. 196, 409-430.

Kheisin, D. Y. 1963 Moving load on an elastic plate which floats on the surface of an ideal fluid (in Russian). Ivz. AN SSSR, OTN, Mekh i Mashinostroenie 178.

LAGET, O. \& Dias, F. 1997 Numerical computation of capillary-gravity interfacial solitary waves. J. Fluid Mech. 349, 221-251.

Miles, J. \& Sneyd, A. D. 2003 The response of a floating ice sheet to an accelerating line load. $J$. Fluid Mech. 497, 435-439.

Milinazzo, F., Shinbrot, M. \& Evans, N. W. 1995 A mathematical analysis of the steady response of floating ice to the uniform motion of a rectangular load. J. Fluid Mech. 287, 173-197.

Nugroho, W., Wang, K., Hosking, R. \& Milinazzo, F. 1999 Time-dependent response of a floating flexible plate to an impulsively started steadily moving load. J. Fluid Mech. 381, 337-355.

Parau, E. \& Dias, F. 2002 Nonlinear effects in the response of a floating ice plate to a moving load. J. Fluid Mech. 460, 281-305.

Peake, N. 2001 Nonlinear stability of a fluid-loaded elastic plate with mean flow. J. Fluid Mech. 434, 101-118.

Schulkes, R. M. S. M. \& SNeYD, A. D. 1988 Time-dependent response of floating ice to a steadily moving load. J. Fluid Mech. 186, 25-46. 
Squire, V. A., Hosking, R. J., Kerr, A. D. \& Langhorne, P. J. 1996 Moving Loads on Ice Plates. Kluwer.

Squire, V. A., Robinson, W. H., Langhorne, P. J. \& Haskell, T. G. 1988 Vehicles and aircraft on floating ice. Nature 33, 159-161.

TAKizaWA, T. 1985 Response of a floating sea ice sheet to a steadily moving load. J. Geophys. Res. 93, 5100-5112.

TAKIZAWA, T. 1988 Deflection of a floating sea ice sheet induced by a moving load. Cold Regions Sci. Tech. 11, 171-180.

Wang, K., Hosking, R. \& Milinazzo, F. 2004 Time-dependent response of a floating viscoelastic plate to an impulsively started moving load. J. Fluid Mech. 521, 295-317.

West, B. J., Brueckner, K. A., Janda, R. S., Milder, D. M. \& Milton, R. L. 1987 A new numerical method for surface hydrodynamics. J. Geophys. Res. 92 (C11), 11,803-11,824.

Whitham, G. B. 1974 Linear and nonlinear waves. Wiley-Interscience. 